\title{
Tomato (Solanum lycopersicum L.) SIIPT3 and SIIPT4 isopentenyltransferases mediate salt stress response in tomato
}

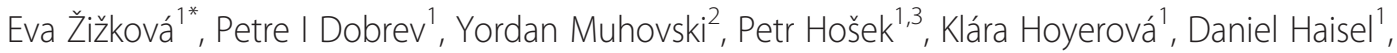 \\ Dagmar Procházková ${ }^{1}$, Stanley Lutts ${ }^{4}$, Václav Motyka ${ }^{1}$ and Imène Hichri ${ }^{4,5^{*}}$
}

\begin{abstract}
Background: Cytokinins (CKs) are involved in response to various environmental cues, including salinity. It has been previously reported that enhancing CK contents improved salt stress tolerance in tomato. However, the underlying mechanisms of CK metabolism and signaling under salt stress conditions remain to be deciphered.

Results: Two tomato isopentenyltransferases, SIIPT3 and SIIPT4, were characterized in tomato and Arabidopsis. Both proteins displayed isopentenyltransferase (IPT) activity in vitro, while their encoding genes exhibited different spatio-temporal expression patterns during tomato plant development. SIIPT3 and SIIPT4 were affected by the endogenous CK status, tightly connected with CKs feedback regulation, as revealed by hormonal treatements. In response to salt stress, SIIPT3 and SIIPT4 were strongly repressed in tomato roots, and differently affected in young and old leaves. SIIPT3 overexpression in tomato resulted in high accumulation of different CK metabolites, following modifications of CK biosynthesis-, signaling- and degradation-gene expression. In addition, 35S:::SIIPT3 tomato plants displayed improved tolerance to salinity consecutive to photosynthetic pigments and $\mathrm{K}^{+} / \mathrm{Na}^{+}$ratio retention. Involvement of SIIPT3 and SIIPT4 in salt stress response was also observed in Arabidopsis ipt3 knock-out complemented plants, through maintenance of CK homeostasis.

Conclusions: SIIPT3 and SIIPT4 are functional IPTs encoded by differently expressed genes, distinctively taking part in the salinity response. The substantial participation of SIIPT3 in CK metabolism during salt stress has been determined in 35S::SIIPT3 tomato transformants, where enhancement of CKs accumulation significantly improved plant tolerance to salinity, underlining the importance of this phytohormone in stress response.
\end{abstract}

Keywords: Cytokinin, Isopentenyltransferase, Salt stress, SIIPT3, SIIPT4, Tomato

\section{Background}

Tomato (Solanum lycopersicum L.) is one of the most popular vegetable species grown world-wide because of its edible fruit and agronomic importance. Nevertheless, its production is frequently threatened by environmental stresses such as drought and salinity. High salt concentration reduces tomato germination, leaf number and area, slows down shoot and root growth and increases

\footnotetext{
*Correspondence: zizkovae@ueb.cas.cz; imene.hichri@sophia.inra.fr ${ }^{1}$ Institute of Experimental Botany, Academy of Sciences of the Czech Republic, Prague 165 02, Czech Republic

${ }^{4}$ Groupe de Recherche en Physiologie Végétale (GRPV), Earth and Life Institute - Agronomy (ELI-A), Université catholique de Louvain (UCL), Louvain-la-Neuve 1348, Belgium

Full list of author information is available at the end of the article
}

root/shoot ratio, induces leaf senescence, and ultimately impairs crop production [1]. Salinity imposes both an osmotic stress due to water shortage, followed by an ionic stress resulting from disproportionate nutrients accumulation and an augmentation of toxic ion concentrations, such as sodium [2,3]. Modifications of plant physiological processes following salinization are partly attributed to alterations in phytohormone metabolism. This may be true especially for cytokinins (CKs). Indeed, under long-term salt stress the level of bioactive CKs in tomato plants was reduced down to $50 \%$ both in roots and leaves $[2,4,5]$.

The ability to control cell division and differentiation, root growth, leaf senescence, apical dominance, branching, 
flower and seed development as well as germination and nutrients uptake into sink organs defines CKs as essential regulatory substances in plants [6]. Chemically, CKs are derivatives of adenine substituted at the $N^{6}$ position either with isoprenoid or an aromatic side chain. Both isoprenoid and aromatic CKs are present in plants in bioactive forms, as free bases and corresponding nucleosides and nucleotides, and in non-active or storage forms as conjugates with glucose (CK-O- and CK- $N$-glucosides) for instance. The typical representatives of isoprenoid CKs are derivatives of trans-zeatin $(t \mathrm{Z})$, cis-zeatin $(c \mathrm{Z})$, dihydrozeatin (DHZ) and $N^{6}-\left(\Delta^{2}\right.$-isopentenyl)adenine (iP), occuring throughout the plant kingdom $[7,8]$. The first step of CK biosynthesis is performed by isopentenyltransferases (IPTs). Plant IPTs belong to multigenic families, chiefly described in Arabidopsis (AtIPT1-AtIPT9) [9,10] and in maize, for instance [11]. Recently, IPT genes have also been identified in tomato (SIIPT1-SIIPT6), and their expression patterns specifically investigated during fruit set and development [12]. IPTs catalyze the transfer of the isoprenoid moiety from precursors dimethylallyl diphosphate (DMAPP) or (E)-4-hydroxy-3-methyl-but-2-enyl diphosphate (HMBDP) to adenine nucleotide forms (AMP, ADP, ATP) $[9,10]$. Modulation of CK contents through modification of IPT expression strongly impacts plants development. Indeed, while overexpression of IPT in plants resulted in faster shoot formation, shorter internodes, loss of apical dominance, delay of leaf senescence, higher photosynthetic rates and accumulation of $t \mathrm{Z}$ and its riboside [13-16], Arabidopsis ipt deficient plants showed strong inhibition of shoot growth, elongation of primary and lateral roots, and reduction of $t \mathrm{Z}$ and iP contents [17].

Recent progress in genetic engineering of CKs enabled to control plant CK contents, affected plant traits, increased yield production and improved plant adaptation to enviromental stresses such as salinity [18-20]. Indeed, responsiveness of Arabidopsis IPT genes to salt stress has been demonstrated by transcriptome analyses, where upregulation of AtIPT1, AtIPT2 and AtIPT8 and downregulation of AtIPT3, AtIPT5, AtIPT7 and AtIPT9 were reported [21]. Regulation of IPT expression through inducible promoter in tomato roots improved tolerance to salt stress and led to higher yield compared to the wildtype plants $[3,4]$. Similarly, plant salinity tolerance was enhanced in transgenic cotton expressing IPT under control of a cysteine proteinase (Ghcysp) promoter, delaying the salt-induced senescence of leaves [22]. Positive correlation between IPT expression and salt resistance has been demonstrated in transgenic tobacco by introducing Agrobacterium tumefaciens IPT under control of the stress-inducible promoter $r d 29 A$ as well [23].

In addition to CK biosynthesis, CK signaling steps are also critical for plant response to salinity. CKs are perceived at the plasma membrane by specific receptors, and the signal is transduced via type-B Arabidopsis Response Regulators (ARRs) controlling transcription of type-A ARRs, which act as negative feedback regulators of CK signaling [24]. ARR transcription factors respond in different ways to salt stress and were reported to regulate sodium accumulation in Arabidopsis [25]. Moreover, a transcriptome analysis of Arabidopsis CK deficient ipt1,3,5,7 mutants exposed to salinity revealed the importance of CK regulation on stress-responsive signaling pathways, even under normal conditions of growth [21].

Although CKs assume crucial functions in tomato salt tolerance, data concerning the underlying molecular cues involved in this process remain unclear. To better understand salt impact on CK metabolism in tomato, a thorough characterization of genes coding for IPTs and their response to salinity is required. The present study reports the functional characterization of two tomato IPT encoding genes, SIIPT3 and SIIPT4, both in vitro and in planta. Spatio-temporal expression profiles during plant development, enzymatic activity of both proteins and their involvement in CK biosynthesis were determined. Likewise, SIIPT3 and SIIPT4 participation in response to salt stress was investigated in Arabidopsis and tomato.

\section{Results}

\section{Identification of two putative non-redundant tomato} isopentenyltransferases

To identify IPT encoding genes in tomato, Arabidopsis IPT1-9 coding sequences were used to screen the tomato genome database [26] (www.solgenomics.net). Among the selected expressed sequence tags (ESTs), two were apparently encoding full length proteins and could be amplified by PCR. For the first gene (GenBank accession JF423320), a 1122 bp coding sequence was cloned, including a 990 bp open reading frame (ORF) defining a 329 amino acid (aa) polypeptide of $37.5 \mathrm{kDa}$ (Figure 1A). This gene was recently annotated as SIIPT3 [12]. For the second gene (GenBank accession JF433930), a 1073 bp coding sequence was amplified, exhibiting a 972 bp ORF predicted to encode a 323 a protein of $37.1 \mathrm{kDa}$. This gene was classified as SIIPT4 [12].

SIIPT3 is located on tomato chromosome 1, while SIIPT4 is situated on chromosome 9. In addition, SIIPT3 includes a $293 \mathrm{bp}$ intron localized in the 5' UTR region. SIIPT3 and SIIPT4 display 52\% identity and $72 \%$ similarity. Alignment of SIIPT3 and SIIPT4 proteins with Arabidopsis IPT3 and IPT5 indicates that the IPT catalytic domain represents almost the entirety of the proteins, with exception of the $\mathrm{N}$ - and C-termini (Figure 1A). A phylogenetic analysis of SIIPT3 and SIIPT4 and nineteen additional orthologs belonging to different species was constructed by means of the neighbour-joining method using full-length amino acid sequences (Figure 1B). It 


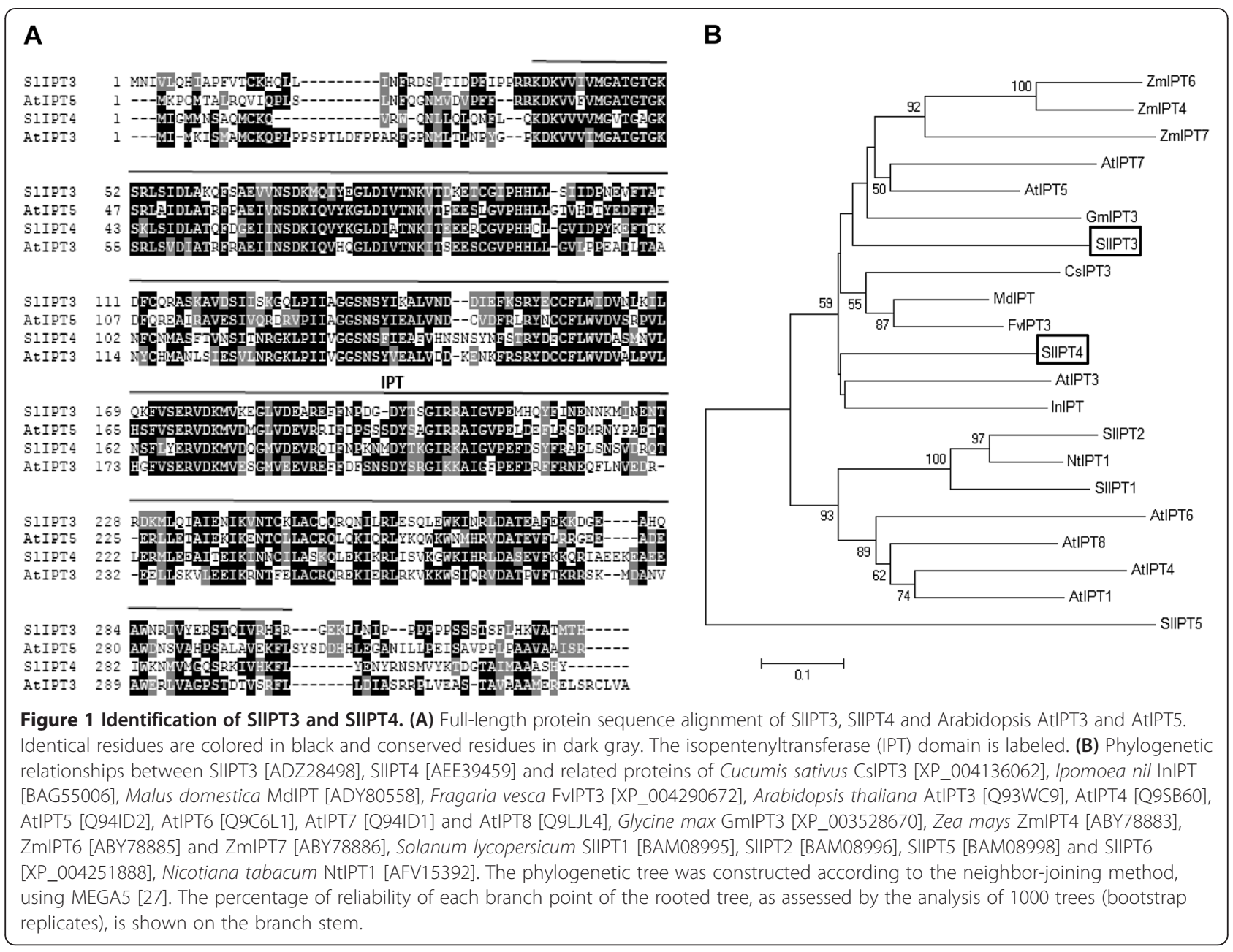

indicated that SIIPT3 mainly clusters with soybean GmIPT3 and Arabidopsis AtIPT5, while SIIPT4 clusters with Arabidopsis AtIPT3 and Japanese Morning Glory InIPT. Except from Arabidopsis and maize, full characterization of IPT enzymes in other plant species is missing.

\section{Tomato SIIPT3 and SIIPT4 show isopentenyltransferase activity in vitro}

To examine the ability of SIIPT3 and SIIPT4 to catalyze CK biosynthesis, a functional analysis of both proteins was carried out. As SIIPT3 and SIIPT4 are highly insoluble when produced in bacteria, both proteins were produced in vitro. The enzymatic activity of SIIPT3 and SIIPT4 was determined using in vitro assays based on conversion of radiolabeled adenylated substrates $\left(\left[{ }^{3} \mathrm{H}\right]\right.$ AMP, $\left[{ }^{3} \mathrm{H}\right]$ ADP or $\left.\left[{ }^{3} \mathrm{H}\right] \mathrm{ATP}\right)$ in the presence of DMAPP to isopentenylated products. Both enzymes were able to convert tritium-labeled AMP/ADP/ATP to the corresponding $\mathrm{iPRMP} / \mathrm{iPRDP} / \mathrm{iPRTP}$ in time-dependent manner (Figure 2A and $\mathrm{B}$ ). The prevailing metabolites formed by both SIIPT3 and SIIPT4 were iPRMP and
iPRDP, with almost 10-fold higher catalytic activity shown by SIIPT4 compared to SIIPT3. These results clearly demonstrate that SIIPT3 and SlIPT4 display CK biosynthetic activity in in vitro conditions.

\section{Subcellular localization of SIIPT3 and SIIPT4}

An analysis of the deduced SIIPT3 and SIIPT4 sequences revealed the presence of chloroplast transit peptides [28] (pSORT, http://psort.ims.u-tokyo.ac.jp/). To investigate protein subcellular localization, SIIPT3 and SIIPT4 cDNA were fused at their $\mathrm{C}$-terminal end to green fluorescent protein (GFP) coding sequence, placed under cauliflower mosaic virus $35 S$ promoter and used to agro-infiltrate tomato leaves. As observed in Figure $3\left(\mathrm{a}, \mathrm{a}^{\mathrm{a}}\right)$, despite a strong auto-fluorescence, SIIPT4 showed localization predominantly in chloroplasts and in the cytosolic area surrounding the chloroplast. Indeed, GFP fluorescence colocalized with chlorophyll fluorescence in this cellular compartment. In contrast, free GFP was restricted to the cytoplasm (Figure 3 $\left(\mathrm{b}, \mathrm{b}^{\mathrm{c}}\right)$ ), while no fluorescence at $520 \mathrm{~nm}$ (GFP emission wavelength) was detected in untransformed tomato leaves (Figure $3\left(\mathrm{c}, \mathrm{c}^{\mathrm{c}}\right)$ ). Despite several attempts, we could not 


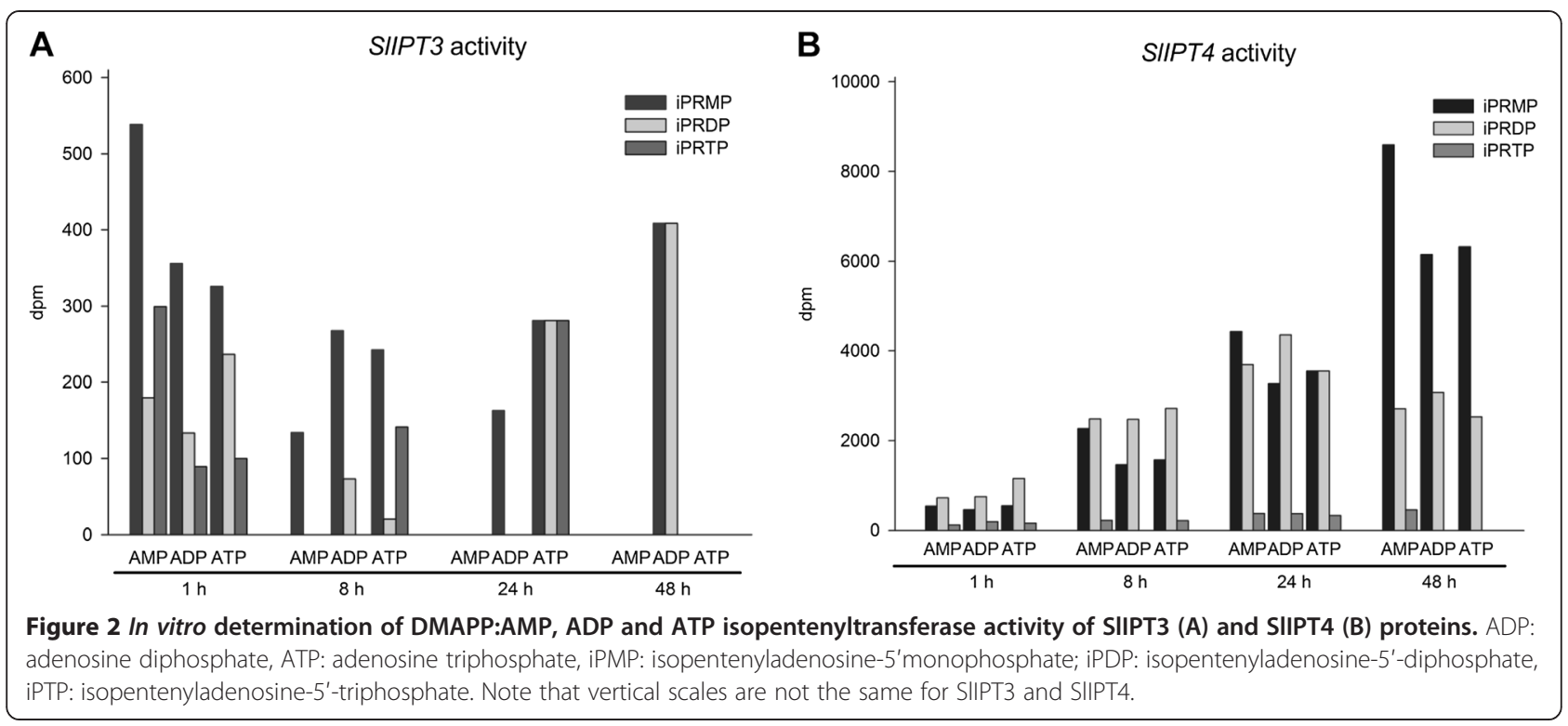

establish subcellular localization for SIIPT3, and no GFP signal of the recombinant protein could be detected in transformed tomato cells. These data show that at least SIIPT4 is localized in the chloroplast and in the cytosolic area surrounding chloroplasts.

\section{Characterization of SIIPT3 and SIIPT4 in Arabidopsis ipt3 mutants}

In parallel, tomato SIIPT3 and SIIPT4 were functionally characterized in planta via complementation of
Arabidopsis ipt3 knock-out (ipt3 KO) mutants. Five days after sowing (DAS), the Arabidopsis wild-type (WT) plants, ipt3 $\mathrm{KO}$ mutants and Arabidopsis ipt3 KO complemented plants showed a similar phenotype on half-strength MS medium (Additional file 1). We thus suggested to determine the function of SIIPT3 and SIIPT4 in Arabidopsis complemented ipt3 KO mutants grown on salt medium and to follow their response to salinity. Based on the highest phenotype discrimination, $100 \mathrm{mM} \mathrm{NaCl}$ medium was used for further testing of
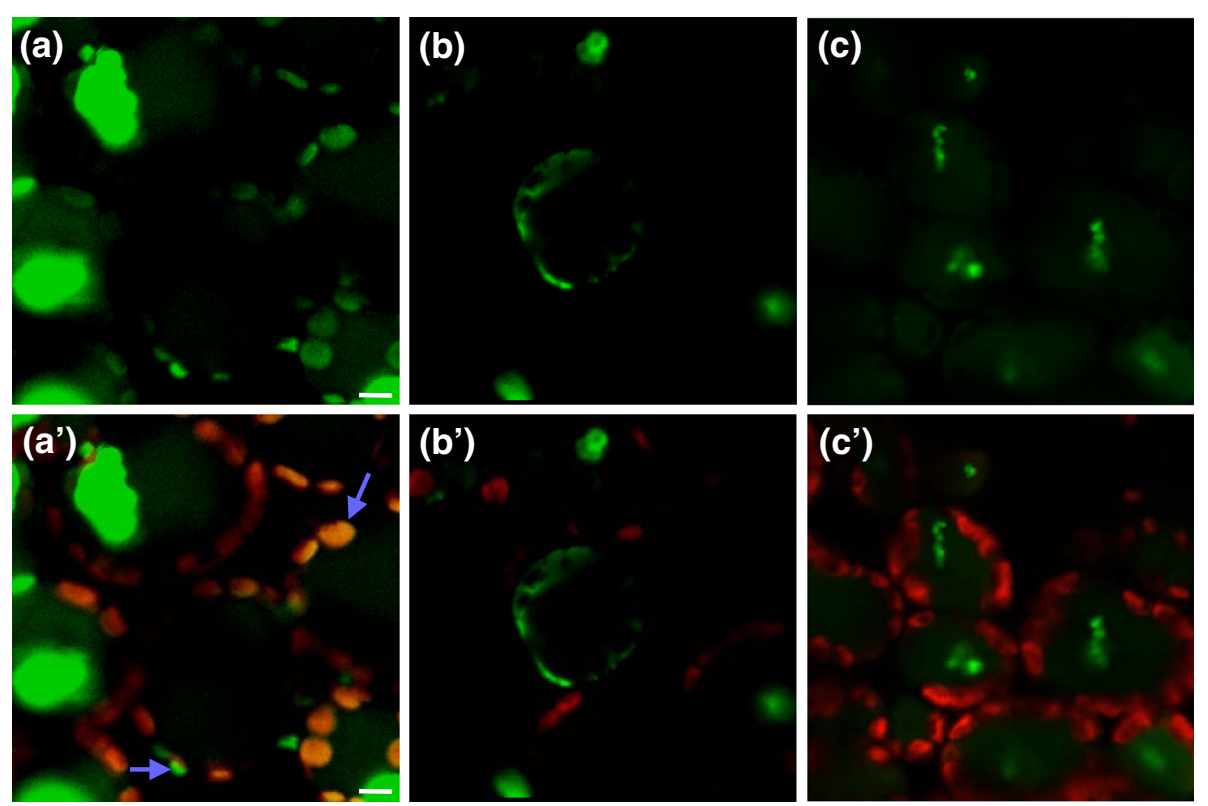

Figure 3 Subcellular localization of SIIPT4-GFP by tomato leaves agroinfiltration. $\left(a, a^{\prime}\right)$ SIIPT4-GFP fluorescence and chlorophyll/GFP fluorescence, respectively. (b, b') Control GFP fluorescence and chlorophyll/GFP fluorescence, respectively. (c, $\mathbf{c}^{\prime}$ ) Control untransformed tomato leaves and chlorophyll autofluorescence, respectively. Bar $=4 \mu \mathrm{m}$. 
seed germination and plant survival percentages, primary root elongation, seedlings CKs content and CK-related genes expression profiles.

On the control medium, germination and survival frequencies were not significantly different in between the variants reaching $100 \%$. On the saline medium, seed germination percentage was reduced to $67 \%$ for both lines of Arabidopsis SIIPT3 complemented plants (9AT and 10AT lines), significantly less than that of ipt $3 \mathrm{KO}$ and WT plants (Figure 4A). However, in Arabidopsis SlIPT4 complemented plants (3AT and 5AT lines), seed germination reached $89 \%$ and $90 \%$, respectively, while WT seeds displayed $78 \%$ and ipt $3 \mathrm{KO} 93 \%$ of germination rate, respectively. The same trend was detected for seedlings' survival percentage on $100 \mathrm{mM} \mathrm{NaCl}$ (Figure 4B) (Fisher's exact test with $\mathrm{p} \leq 0.05$ ). Similarly, primary root elongation was estimated by screening 10 DAS Arabidopsis SIIPT3 and SIIPT4 complemented plants (Figure 4C). On control medium, the primary root length among the 9AT and 10AT lines significantly differed compared to WT while on the salt-containing medium, no significant changes between the SIIPT3 complemented and WT plants were observed. Arabidopsis plants complemented with SIIPT4 showed significantly longer primary root $(3.77 \pm 0.41$ and $3.85 \pm 0.8 \mathrm{~cm}$ for $3 \mathrm{AT}$ and $5 \mathrm{AT}$, respectively) than WT $(3.35 \pm 0.58 \mathrm{~cm})$ on control medium, while antagonistic effects were detected on salt-containing medium (Figure 4C, Mann-Whitney U Test with $\mathrm{p} \leq 0.05$ ). These results demonstrate that both tomato IPT enzymes are functional in heterologous system. However, in reaction to salt stress the SIIPT4 complemented plants displayed the same characteristics as WT in germination and survival assays, while both of the SIIPT3 complemented lines differed in their response to salinity.
A

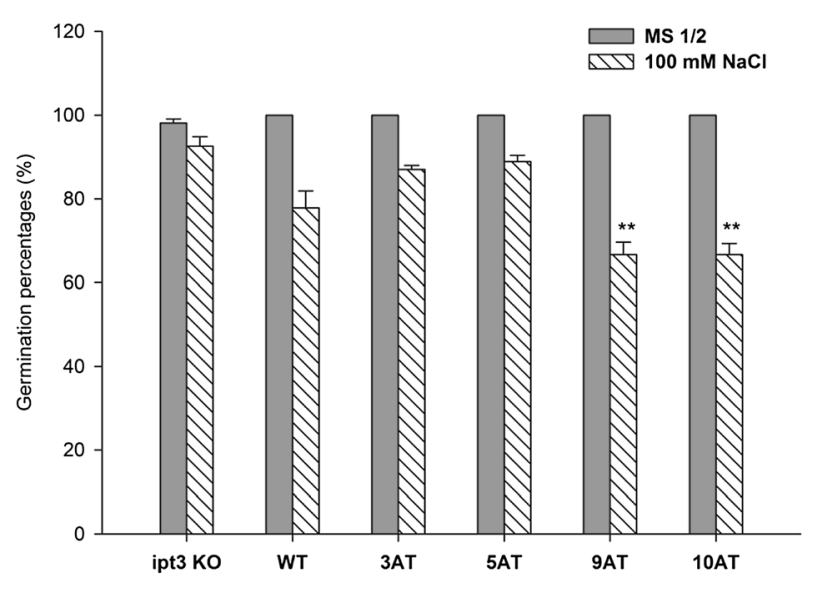

C

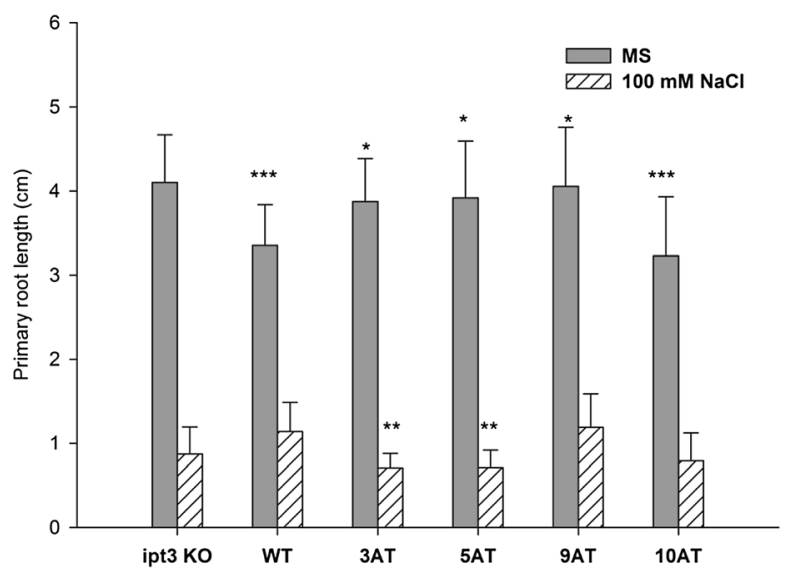

B

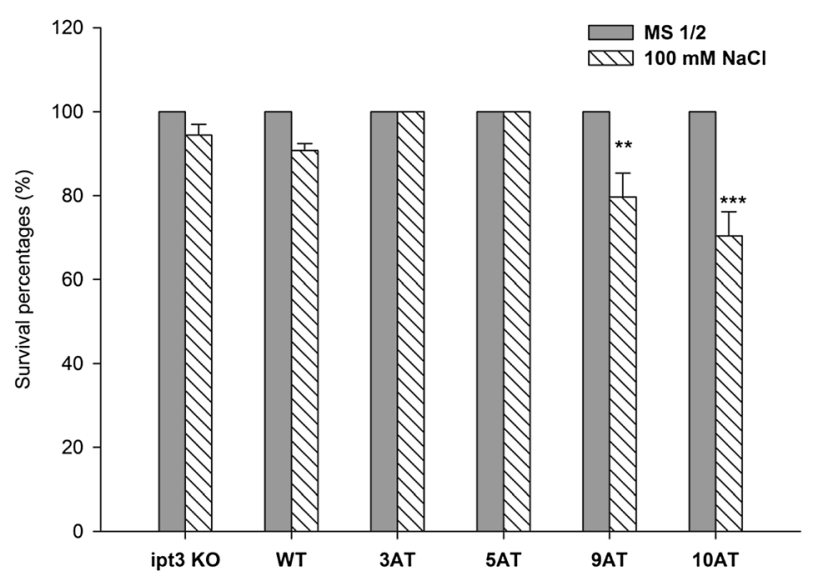

Figure 4 Salinity response of SIIPT3 and SIIPT4 in Arabidopsis. Germination percentage (A) and survival percentage (B) of SIIPT4 (lines 3AT and 5AT) and SIIPT3 (lines 9AT and 10AT) Arabidopsis ipt3 complemented plants. ** significantly lower germination and survival percentages compared to ipt3 KO. ***significantly lower survival percentages compared to WT and ipt3 KO. (C) Primary root length of Arabidopsis SIIPT3 and SIIPT4 complemented plants. * significantly longer primary root compared to WT. ${ }^{* *}$ significantly shorter primary root compared to WT. ***significantly shorter primary root compared to ipt3 KO plants (Mann-Whitney U Test with $p \leq 0.05$ ). 
To assess whether salt treatment affected CK metabolism in Arabidopsis SIIPT3 and SIIPT4 complemented plants, CK contents and gene expression profiles of CK biosynthetic genes were investigated on control and salt medium $(100 \mathrm{mM} \mathrm{NaCl})$. Generally, in both types of media, the total content of CKs of Arabidopsis SIIPT3 and SIIPT4 complemented plants exceeded that of ipt3 $\mathrm{KO}$ plants. However, in comparison to WT plants, Arabidopsis ipt3 complemented plants showed lower CK concentrations in transgenic lines with exception of the 9AT line on salt medium (Figure 5A). Proportionally, CK- $N$-glucosides accumulated strongly in all tested variants (149.01 - 254.81 $\mathrm{pmol} / \mathrm{g} \mathrm{FW}$ ), with iP7G as the predominant metabolite regardless of growth medium (Additional file 2 and Additional file 3). Salt treatment substantially increased the levels of $\mathrm{CK}$ free bases and ribosides, while $t Z$-type CKs were mainly enhanced among the CK groups (Figure 5B).

The expression profiles of CK biosynthetic genes were investigated in Arabidopsis seedlings. No expression in vegetative phase of development was detected for AtIPT4, AtIPT6, and AtIPT8, as had been previously observed [17]. On the control medium, complemented plants showed differential expression of the remaining IPT genes compared to WT or ipt $3 \mathrm{KO}$ plants. AtIPT2 and AtIPT5 were repressed in Arabidopsis SIIPT3 complemented

\section{A}

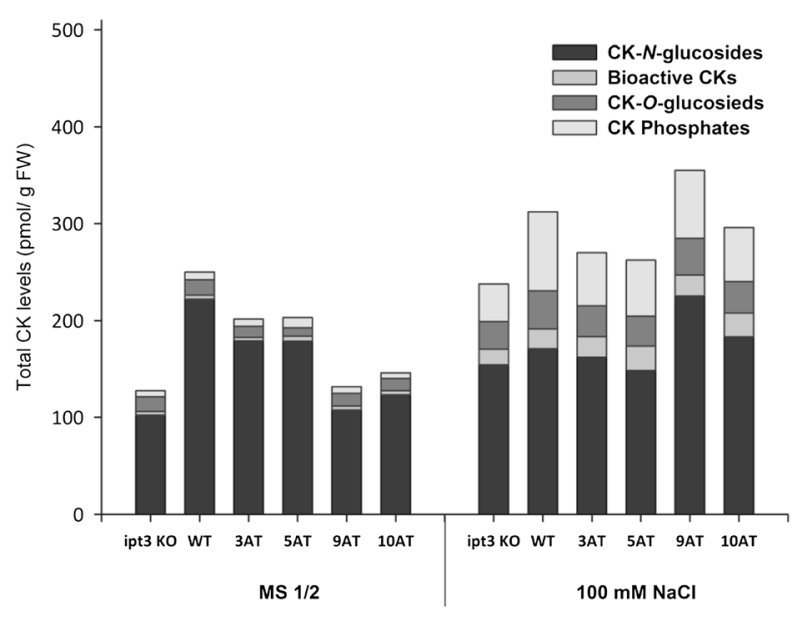

C

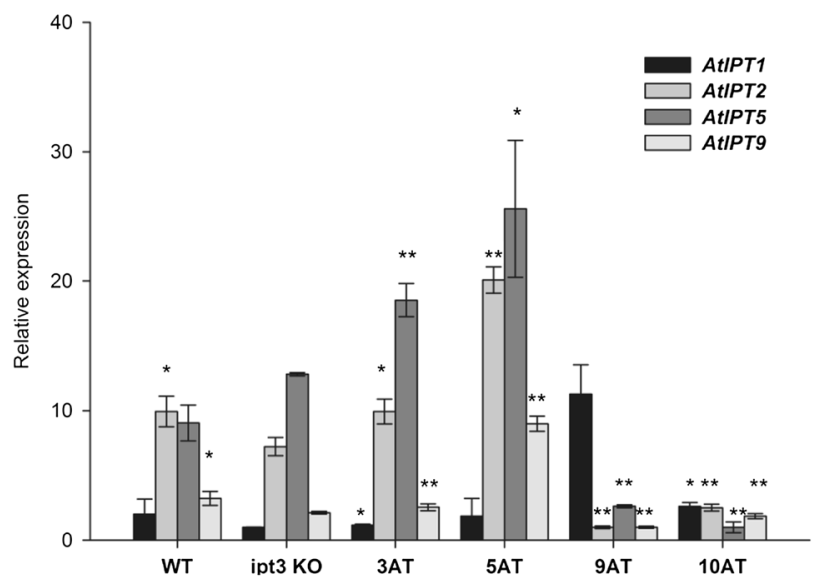

B

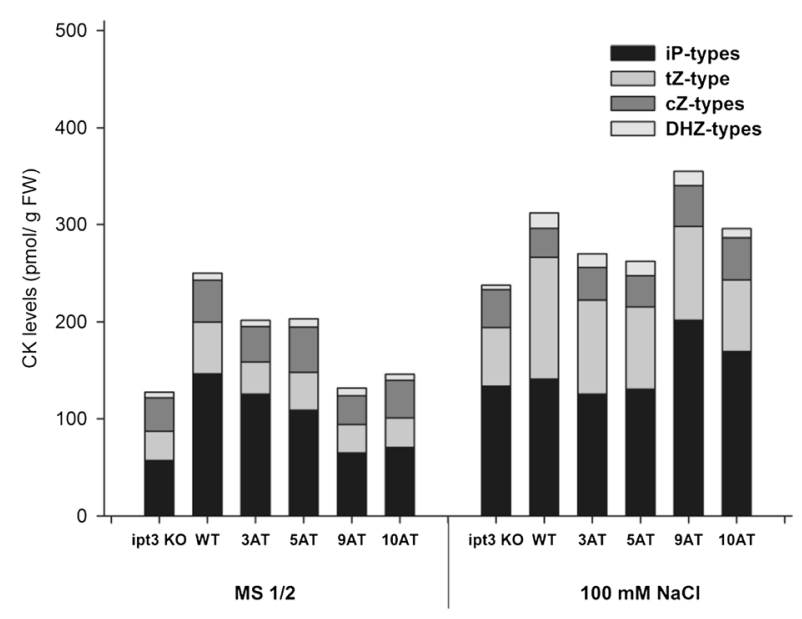

D

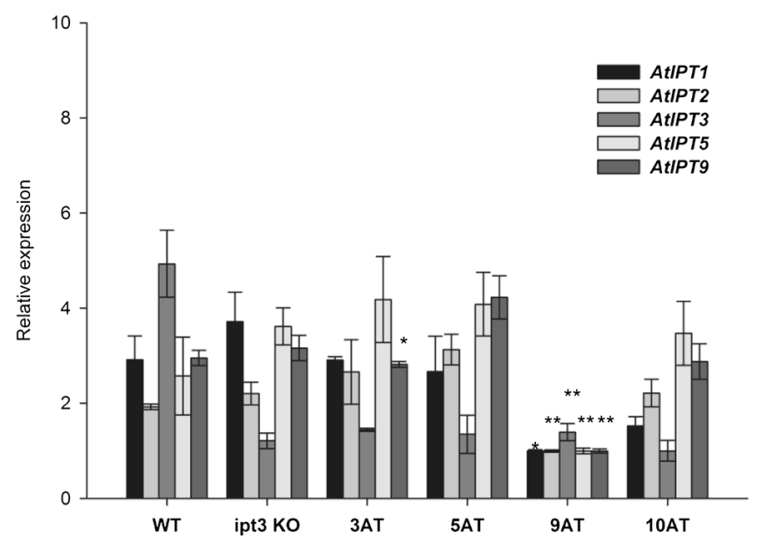

Figure 5 CKs accumulation in Arabidopsis ipt3 complemented plants. Total endogenous CK levels of SIIPT4 (lines 3AT and 5AT) and SIIPT3 (lines 9AT and 10AT) ipt3 KO complemented plants divided according to the chemical structure (A) and contents of bioactive CKs (B). Relative expression of CK biosynthetic genes in Arabidopsis ipt3 KO mutants complemented plants cultivated on the control medium (C) and on the salt-containing (100 mM NaCl) medium (D). Actin and EF1 were used as internal controls for normalization of AtIPT transcript levels. Data represent means and SD of two replicates. * ${ }^{3}$ tatistically significant difference from ipt3 KO (unpaired two-tailed Student's $t$-test, $p \leq 0.05$ ). **statistically significant difference from ipt3 KO after Šidák correction for testing of multiple lines (multiple unpaired two-tailed Student's $t$-test, overall $a=0.05$, individual $p \leq 0.01$ ). 
plants, while their expression increased in Arabidopsis SIIPT4 complemented plants, in comparison with WT or ipt3 KO plants (Figure 5C). Salinity strongly downregulated AtIPT2 and AtIPT5 expression, while it increased expression of AtIPT3 in WT, ipt $3 \mathrm{KO}$ and SlIPT4 complemented plants (Figure 5D). In SlIPT3 complemented plants, genes were differentially regulated depending on the transgenic line considered (9AT or 10AT). These results demonstrate that salinity differently affects IPT genes expression and CK status in Arabidopsis, and that SIIPT3 and SIIPT4 show distinct regulatory mechanisms in heterologous system. Therefore we propose that Arabidopsis plants respond to salt stress by the elevation of CK levels, with predominant accumulation of $\mathrm{N}$-glucosides suggesting overabundance of CKs that is followed by downregulation of some IPT encoding genes to maintain CK homeostasis.

\section{SIIPT3 and SIIPT4 are differently expressed in tomato organs and participate in cytokinin homeostasis}

The expression patterns of SIIPT3 and SIIPT4 in various tomato vegetative and reproductive organs from plants cultivated in the greenhouse under normal growth conditions, including three stages of fruit development and ripening, were investigated by qRT-PCR (Figure 6A and B). In general, SIIPT3 transcripts were much more abundant than SIIPT4 ones in all the tested organs. Both genes were expressed in young and old leaves, roots and stems of tomato plants. In vegetative tissues, SIIPT3 transcripts were weakly detected in young leaves but highly accumulated in

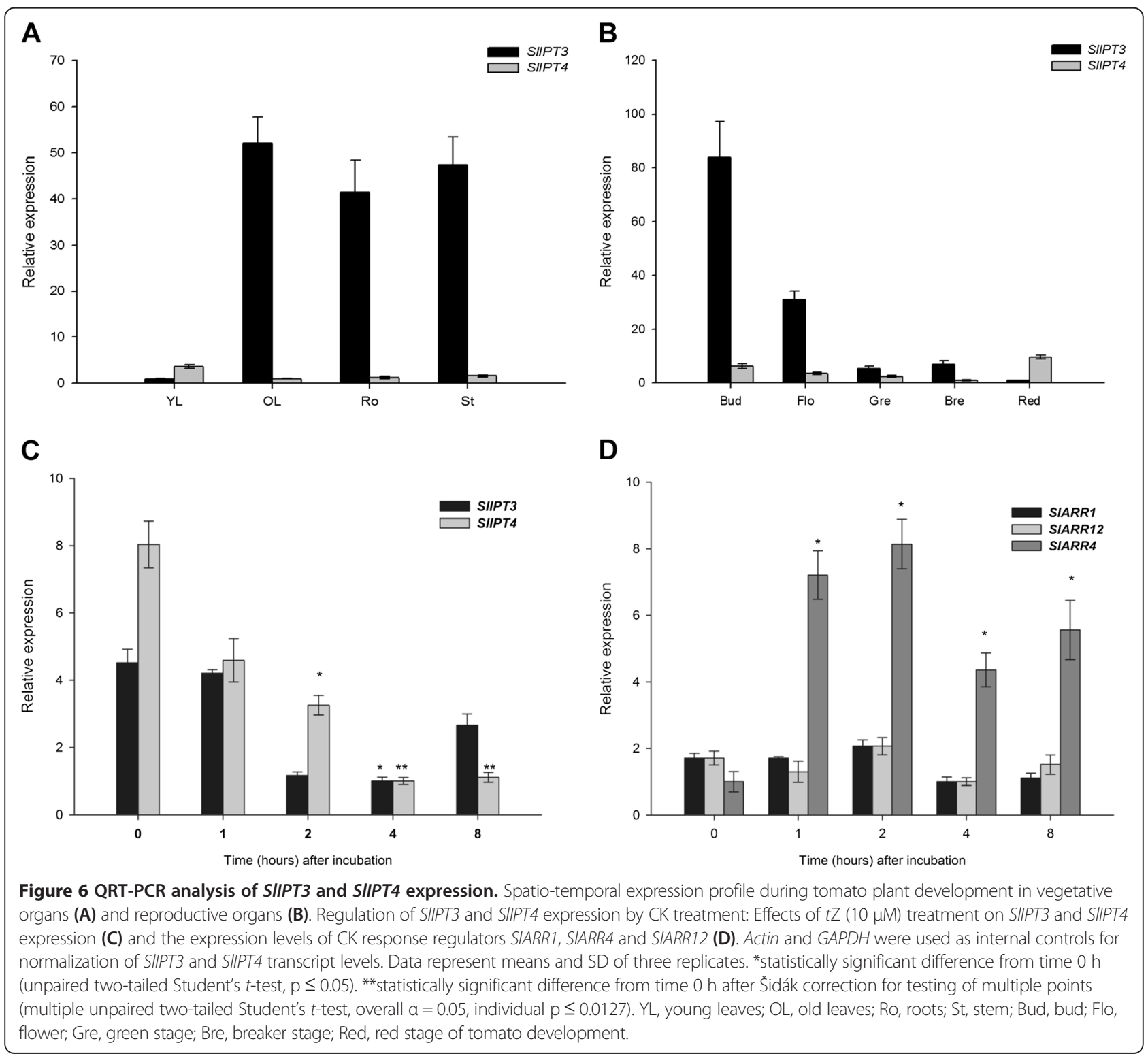


stems, roots and old leaves. In contrast, SIIPT4 was preferentially expressed in young leaves (Figure 6A). In reproductive tissues, SIIPT3 transcripts were abundant in buds and flowers, then rapidly decreased during tomato fruit maturation with higher levels of transcripts in the breaker stage. SlIPT4 was preferentially expressed in buds, then continuously declined until the breaker stage and reached its peak expression at the red stage of tomato fruit (Figure 6B). Taken together, these data indicate that SIIPT3 and SIIPT4 display distinct expression profiles during tomato development.

In order to clarify a putative feedback regulation by CKs on SIIPT3 and SIIPT4 expression, WT tomato roots from hydroponic plants were treated with $t \mathrm{Z}$. CK treatment gradually repressed SIIPT3 (up to 4-fold) and SIIPT4 (up to 8-fold) expression, the inhibitory effect being most noticeable $4 \mathrm{~h}$ after treatment onset. However, SIIPT3 transcripts then started to accumulate again, which was not the case for SIIPT4 (Figure 6C). Likewise, the exogenous application of $t Z$ strongly induced expression of the CK negative-feedback ARR type-A SlARR4, in contrast to its more discrete effect on ARR type-B SIARR1 and SIARR12 (Figure 6D). Altogether, these data suggest a regulation of SIIPT3 and SIIPT4 transcripts accumulation through plant endogenous CK status, in addition to the feedback regulation via the CK signaling pathway.

\section{Overexpression of SIIPT3 strongly impacts tomato phenotype and cytokinin status}

To understand the impact of SIIPT3 and SIIPT4 overexpression on CK metabolism and verify the enzymes activity in planta, we attempted to generate transgenic lines constitutively expressing SIIPT3 or SIIPT4. Several $35 S$ ::SlIPT3 transgenic lines were able to develop and survive repotting into soil, while no $35 S$ ::SLIPT4 tomato plants could be regenerated from transgenic calli in vitro. For further characterization, three 35 S::SIIPT3 transgenic lines (L6, L8, L9) were selected, with transgene expression in leaves increasing up to 14-fold (L9) compared to WT (Figure 7A).

The phenotype of 35S::SIIPT3 tomatoes substantially differed from that of the WT plants. SIIPT3 overexpression induced plant dwarfism, release from apical dominance with a branchy phenotype, thick and ligneous stems, thicker leaves with punctual shape modification and shorter internodes (Figure 8A (a-d)). A significant yellowing of leaves, as well as accumulation of anthocyanin, was also observed. Additionally, despite inflorescence formation, no flowers were visible in most of SIIPT3 transgenic lines (Figure 8A (f)) compared to WT plants (Figure 8A (g)). Only a single transgenic line (L1) showing the weakest phenotype (Additional file 4) was able to develop normal fruits containing seeds.
Generally, during several months of cultivation, fruit formation was either significantly reduced or completely aborted. When fruits did develop, ripening was incomplete in transgenic plants. Taken together, these observations indicated that constitutive overexpression of SIIPT3 strongly affects tomato plant development, morphogenesis and reproduction.

The strong effects of SIIPT3 overexpression on tomato phenotype were simultaneously reflected in CK metabolism. The total CK contents extracted from young and fully expanded tomato leaves increased up to 12-fold in 35S::SlIPT3 lines (Figure 8B, Additional file 5) compared to WT plants. Proportionally, CK- $\mathrm{N}$-glucosides represented the main group of CKs, especially in $35 S::$ SIIPT3 lines, with iP7-glucoside (iP7G) being the predominant metabolite (Figure 8B, Additional file 5). In addition, the highest concentration of $t \mathrm{Z}$ was observed in WT plants (Figure 8C, Additional file 5). Interestingly, the CK storage forms (CK-O-glucosides) only slightly increased (up to 2.6-fold) in all transformants (Figure 8B). These results show that SIIPT3 overexpression leads to a dramatic increase in CKs, particularly to the enhanced formation of iP7G throughout N7-glucosylation pathway, which seems to be the major metabolic pathway for bioactive CKs inactivation. Indeed, no significant differences in CK oxidase/dehydrogenase (CKX) activity were found between the control and transgenic tomatoes (Additional file 6).

\section{SIIPT3 overexpression modifies cytokinin-related genes expression and plant tolerance to salinity}

To elucidate the role of CK biosynthesis (IPT), signaling (ARRs) and catabolism (CKX) related genes in the response to SIIPT3 overexpression in tomatoes, the expression patterns of corresponding genes were determined by qRT-PCR in young leaves of transgenic tomatoes and WT plants. In 35S::SlIPT3 lines, the transcript abundance of SlIPT6 (which encodes a tRNA dimethylallyltransferase) was higher than in WT, whereas lower expression of SIIPT1 was observed in all transgenic lines (Figure 7A). Likewise, upregulation of SIARR4, functioning in feedback regulatory loops of CKs (Figure 7B), and downregulation of CK deactivating genes SlCKX4 and SlCKX5 (Figure 7C) were observed in all transformants, relative to WT plants. Thus, CK-related genes expression analysis revealed upregulation of some genes involved in CK biosynthesis (SIIPT6) and signaling (SIARR4), but downregulation of genes involved in CK degradation pathways (SlCKX4 and SlCKX5).

We hypothesized that the upregulation of some CK metabolic genes and enhancement of CKs content could be elementary prerequisites of tomato salt stress tolerance. As only L1 could successfully reproduce, T2 homozygous transformants from this transgenic line were further analyzed for their tolerance to salinity. For this purpose, L1 and WT lines were cultivated in vitro for 4 weeks on the 


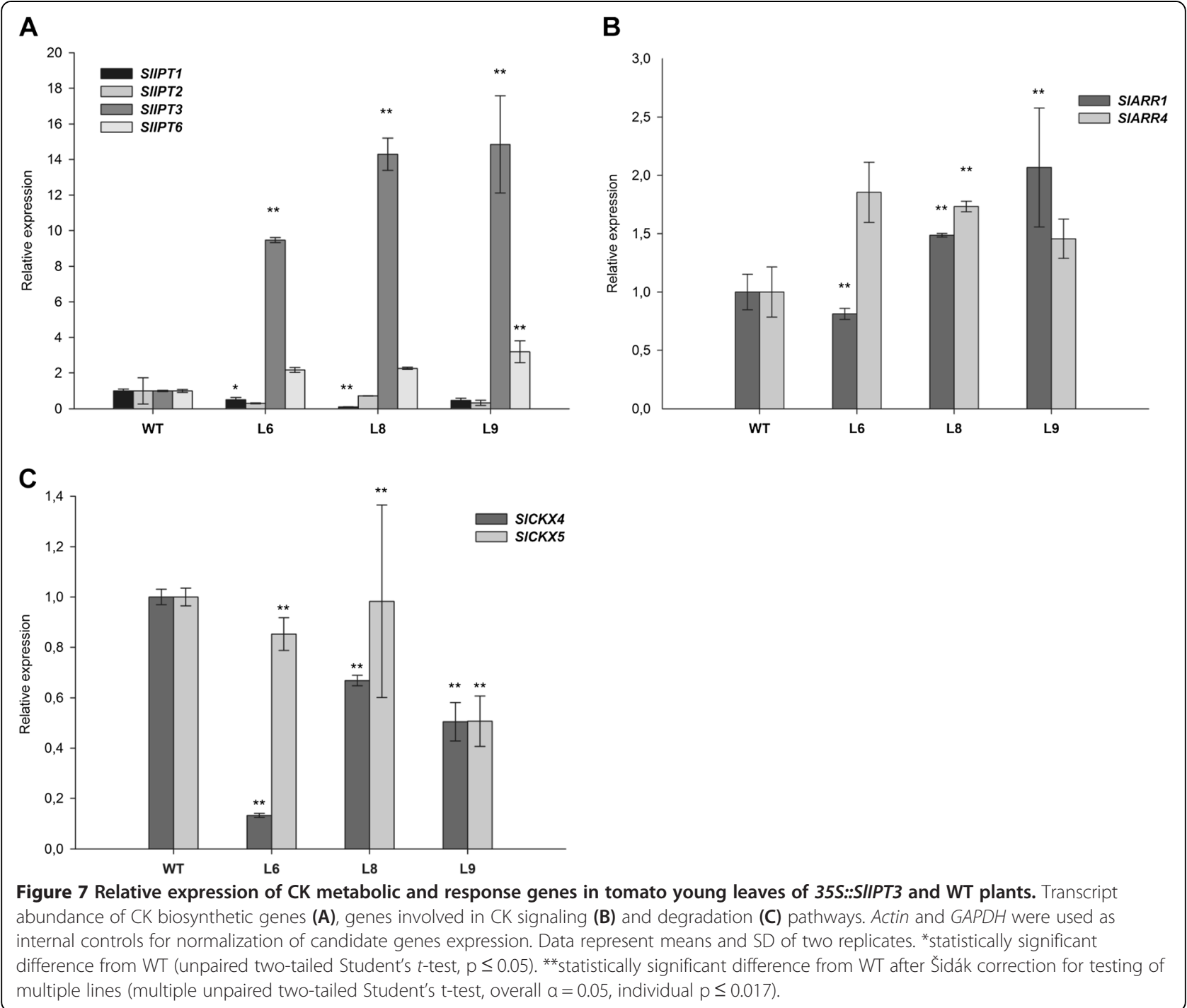

$100 \mathrm{mM} \mathrm{NaCl}$ medium. Compared to WT plants, $35 \mathrm{~S}::$ SIIPT3 L1 plants exhibited a larger shoot with extending leaves, whereas WT plants showed more compact shoot and smaller leaves as a result of limited growth in presence of salt (Figure 9A). Subsequently, the effects of salt stress induced either by $100 \mathrm{mM}$ and $150 \mathrm{mM} \mathrm{NaCl}$ concentration on photosynthesis and nutrients balance were evaluated in WT and L1 by measurements of photosynthetic pigments and macronutrients contents.

In WT plants, chlorophyll $a$ (Chl $a$ ) and chlorophyll $b$ $(\mathrm{Chl} b)$ significantly decreased with the addition of $\mathrm{NaCl}$ into the growth media. In L1 plants, the content of both types of chlorophyll was significantly lower on control medium relative to WT, but remained nearly constant on $\mathrm{NaCl}$ containing media, and a significant decrease in Chl $a$ content was only apparent on $150 \mathrm{mM} \mathrm{NaCl}$ (Figure 9B). Likewise, the levels of carotenoids (Car), especially lutein and $\beta$-carotene displaying photoprotective roles in photosynthesis, exhibited almost identical contents in 35S::SIIPT3 L1 plants regardless of growth media. In contrast, WT plants showed a significant decrease of $\beta$-carotene on both types of $\mathrm{NaCl}$ media, and of lutein on $150 \mathrm{mM} \mathrm{NaCl}$. Increasing contents of Car involved in the xanthophyll cycle, violaxanthin (V), antheraxanthin (A) and zeaxanthin $(\mathrm{Z})$ were as well observed in presence of salt in both WT and transgenic tomatoes, significant for WT at $150 \mathrm{mM} \mathrm{NaCl}$ and 35S::SlIPT3 grown on both concentrations of salt (Figure 9C and Additional file 7).

Shoot sodium $\left(\mathrm{Na}^{+}\right)$, potassium $\left(\mathrm{K}^{+}\right)$, magnesium $\left(\mathrm{Mg}^{2+}\right)$ and calcium $\left(\mathrm{Ca}^{2+}\right)$ concentrations were assessed in plants exposed to 0 (control), $100 \mathrm{mM}$ and $150 \mathrm{mM} \mathrm{NaCl}$ (Figure 9D and E). As observed in Figure 9D, $\mathrm{Na}^{+}$and $\mathrm{K}^{+}$concentrations were similar in WT and L1 under control conditions of growth. Salinity induced $\mathrm{Na}^{+}$accumulation and substantially reduced $\mathrm{K}^{+}$contents in both types of tomato plants. However, $\mathrm{Na}^{+}$accumulated to a lesser 


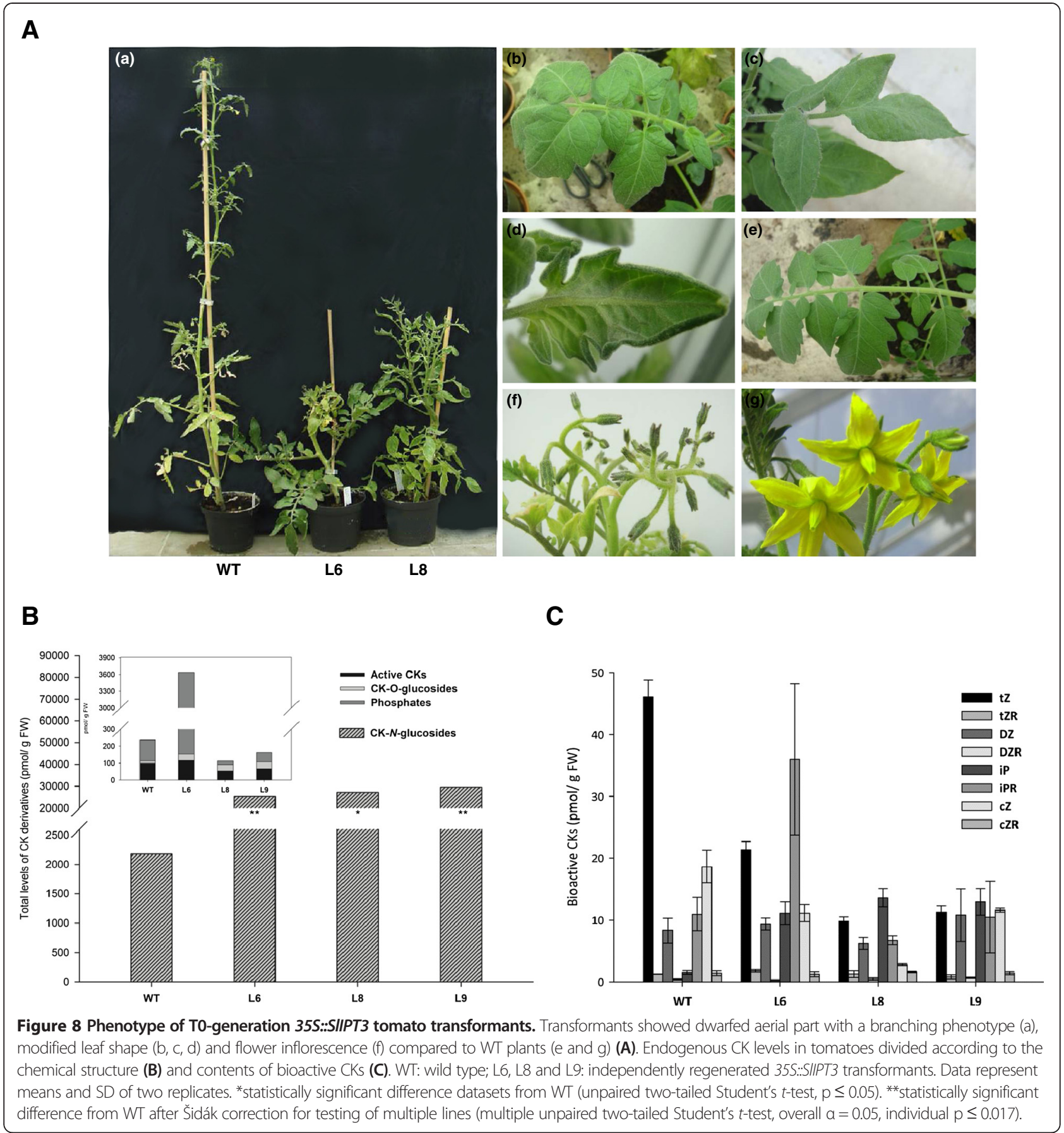

extent in L1 (0.33 and $0.37 \mathrm{mmol} / \mathrm{g}$ DW) than in WT (0.38 and $0.48 \mathrm{mmol} / \mathrm{g} \mathrm{DW})$ on the 100 and $150 \mathrm{mM}$ $\mathrm{NaCl}$ medium, respectively. Similarly, $\mathrm{K}^{+}$concentration was significantly higher in L1 $(0.27$ and $0.22 \mathrm{mmol} / \mathrm{g} \mathrm{DW})$ than in WT $(0.216$ and $0.154 \mathrm{mmol} / \mathrm{g} \mathrm{DW})$ in presence of 100 and $150 \mathrm{mM} \mathrm{NaCl}$, respectively, and these results are also visible by the $\mathrm{K}^{+} / \mathrm{Na}^{+}$ratio, which remained significantly higher in L1 than in WT in salt stress conditions (Figure 9E). Finally, no difference of $\mathrm{Ca}^{2+}$ contents between WT and transgenic plants was noticed, while the $\mathrm{Mg}^{2+}$ concentration was significantly higher in transgenic tomatoes on control and $100 \mathrm{mM} \mathrm{NaCl}$ conditions, compared to WT (Figure 9D).

Overall, plant morphological changes in presence of salt, roughly constant levels of photosynthetic pigments, especially Chl $a$, Chl $b$, lutein and $\beta$-carotene in 35S:: SIIPT3 tomatoes regardless on the growth medium and higher $\mathrm{K}^{+}$contents in presence of $\mathrm{NaCl}$ indicate that SIIPT3 overexpression could raise plant tolerance to salt stress. 


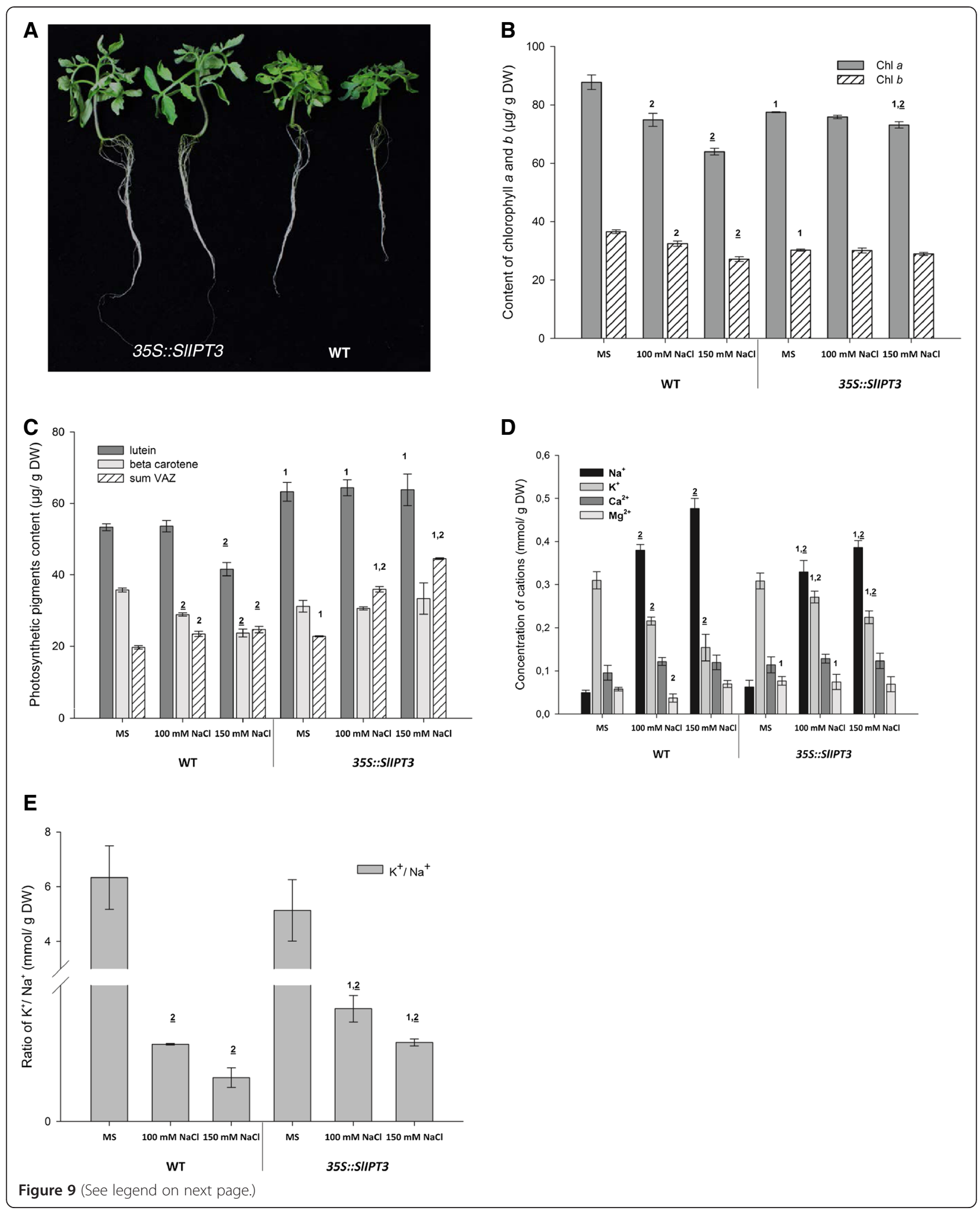


(See figure on previous page.)

Figure 9 Effect of salt stress on WT and 35S::SIIPT3 (L1) tomato plants cultivated on control and salt-containing growth media. (A) Phenotype of WT and 35S::SIIIPT3 (L1) transgenic plants grown 4 weeks on the $100 \mathrm{mM} \mathrm{NaCl}$ medium. Chlorophyll content (B), carotenoid content (C), cation concentration (D) and $\mathrm{K}^{+} / \mathrm{Na}^{+}$ratio (E) in 4 weeks-old tomato developing leaves of WT and trangenic plants. Sum VAZ, sum violaxanthin, antheraxanthin and zeaxanthin. Data represent means and SD of two replicates for photosynthetic pigments while three replicates represent data from cation concentration. ' statistically significant difference from the same treatment of WT (unpaired two-tailed Student's $t$-test, $p \leq 0.05$ ). ${ }^{2}$ statistically significant difference from MS medium within the same line (unpaired two-tailed Student's $t$-test, $p \leq 0.05$ ), ${ }^{2}$ statistically significant difference from MS medium within the same line after Šidák correction for testing of multiple NaCl concentrations (multiple unpaired two-tailed Student's $t$-test, overall $a=0.05$, individual $p \leq 0.0253$.

Both SIIPT3 and SIIPT4 are involved in salt stress response To further understand the participation of SIIPT3 and SIIPT4 in salt stress response, their expression pattern was examined by qRT-PCR in roots, young leaves and old leaves of tomato WT plants exposed to salinity (150
$\mathrm{mM} \mathrm{NaCl}$ ) for $12 \mathrm{~h}$. In roots (Figure 10A), which are the first organ perceiving salinity, SIIPT3 and SIIPT4 shared very similar expression profiles. A rapid decrease in transcript levels of both genes within $2 \mathrm{~h}$ after salt exposure was followed by a very small peak at $8 \mathrm{~h}$ for

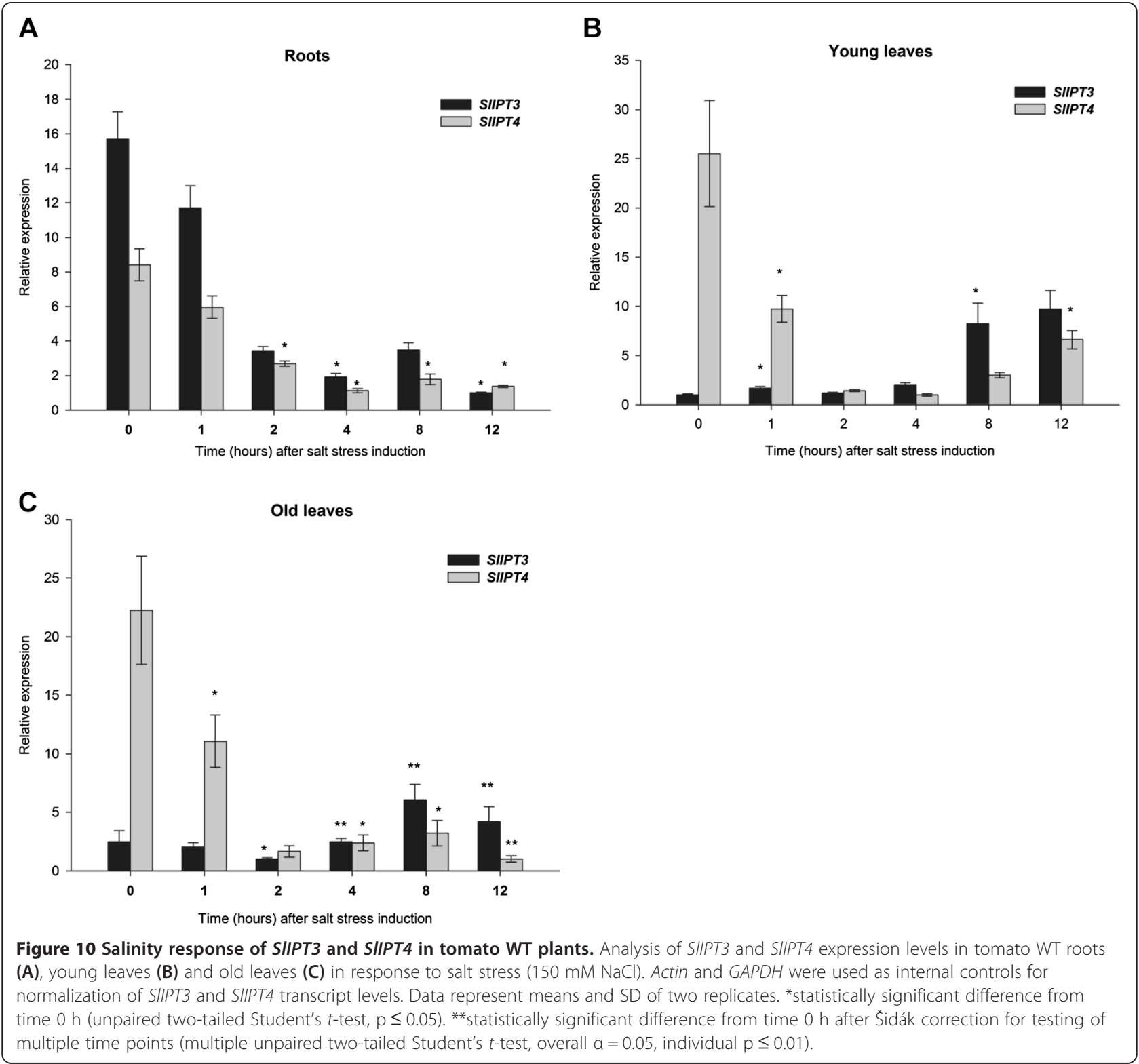


both genes, before a new decline. In young leaves, SIIPT3 and SIIPT4 showed partially divergent transcript accumulation profiles (Figure 10B). Indeed, SIIPT3 transcripts gradually and regularly increased with incubation time. In contrast, SIIPT4 was repressed 2.5-fold as rapidly as $1 \mathrm{~h}$ after stress initiation. This repression seemed transient, however, as a slight increase in SIIPT4 transcripts was visible $8 \mathrm{~h}$ after stress onset (Figure 10B). Similar expression profiles were observed in old leaves (Figure 10C). These results indicate that SIIPT3 and SIIPT4 directly participate in salt stress response in tomato via a differential modulation of their expression, depending on the organ and duration of the stress.

\section{Discussion}

CK homeostasis during plant development and in response to changing environmental conditions is, among other mechanisms, properly maintained by the activity of CK biosynthetic enzymes, IPTs. It was previously reported that root-localized IPT expression essentially improved salt stress tolerance in tomato [3]. However, the genes involved and the mechanisms of CKs regulation under salt stress conditions have not yet been elucidated. Therefore, based on functional characterization of SIIPT3 and SIIPT4 we provide insights into both genes involvement in the response to salt stress in tomato plants.

\section{Tomato SIIPT3 and SIIPT4 are non-redundant functional isopentenyltransferases}

In the present study, we have demonstrated that tomato SIIPT3 and SIIPT4 encode two functional isopentenyltransferases (Figure 2A). Multiple alignment of proteins sequences and phylogenetic analysis revealed that SIIPT3 and SIIPT4 are closely related to Arabidopsis ortholog AtIPT3 and AtIPT5 (Figure 1B), as previously reported [12]. Interestingly, we observed identical chloroplast localization of GFP-tagged SIIPT4 protein in tomato leaf cells (Figure 3), as described in Arabidopsis where AtIPT3 was localized to plastids in roots and leaf cells [29]. These data indicate that SIIPT4 and AtIPT3 partly share similar function and protein subcellular localization. We therefore speculate that SIIPT4, and probably also SIIPT3, due to the presence of a chloroplastic transit peptide as well, may have other comparable regulatory mechanisms for protein chloroplast targeting such as farnesylation for instance, as was demonstrated for AtIPT3 [30].

Distinct spatio-temporal expression profiles of SIIPT3 and SIIPT4 have been observed in tomato vegetative organs and during fruit onset, development and ripening, indicating that these two genes have non-redundant functions (Figure 6A and B). In general, SIIPT4 expression was lower relative to that of SIIPT3 and showed its highest levels in young leaves and red fruits whereas SIIPT3 substantially predominated in the remaining tested organs. Our data are in agreement with a previous report describing very low transcript abundance of SIIPT4 in all tomato tested organs with flowers exhibiting the highest SIIPT4 expression, and a greater accumulation of SIIPT3 transcripts, especially in the very early stages of flower and fruit development [12]. Interestingly, although SIIPT4 is less expressed than SIIPT3 in tomato, SIIPT4 showed ten times greater activity in vitro than SIIPT3, which may explain why no transgenic plants could be regenerated from calli. Indeed, SIIPT4 is probably at the origin of a greater hormonal imbalance than SIIPT3, which may have impaired proper shoot and root systems regeneration.

Differences between SIIPT3 and SIIPT4 in organ expression specificity point out their distinct physiological roles in tomato developmental processes. However, it seems rather difficult to assign a specific role to each IPT during tomato plant development, due to their overlapping functions. Indeed, although AtIPT3 is one of the three most expressed IPT genes in Arabidopsis during the vegetative phase, ipt 3 knock-out mutant exhibited no distinguishable phenotype from WT plants, indicating functional redundancy between the Arabidopsis IPT proteins [17]. Therefore, none of the visible phenotypes or characteristics in SIIPT3 and SIIPT4 complemented Arabidopsis ipt3 knock-out mutants were apparent in comparison to WT plants under control conditions (Additional file 1, Figure 4A and B).

\section{Ectopic expression of SIIPT3 alters tomato phenotype and CK metabolism}

Overexpression of SIIPT3 under $35 S$ CaMV promoter and consequent $\mathrm{CK}$ over-accumulation had extensive negative effects on tomato growth under control conditions (Figure 8), resulting in dwarf phenotype and delayed senescence, substantially limiting formation of tomato generative organs. Only one transgenic line (L1) showing the weakest phenotypic differences from WT was able to produce seeds (Additional file 4). A similar effect of CK overabundance in flowering delay was reported in stably transformed tobacco plants with 35S::MdIPT3a [31]. Likewise, phenotype alterations such as a strong delay in growth, shorter rosettes, serrated leaves and decreased apical dominance were observed in Arabidopsis plants overexpressing maize ZmIPT2 [32]. In contrast, no changes in flowering time were evident in Arabidopsis and tobacco transgenic plants expressing IPT under HSP7O [33], underlining the crucial role of CKs accumulation and homeostasis during flowering.

We found out that constitutive expression of SIIPT3 increased CK production in tomatoes by up to 12-fold relative to WT plants, primarily iP7G (Figure 8B, Additional file 5). Similarly, high concentrations of iP7G in Arabidopsis complemented plants cultivated on both control and salt media suggest that the formation of 
CK-N7-glucosides may be a sufficient pathway for downregulation of bioactive CKs because no significant enhancement of CKX activity was detected in 35S::SlIPT3 tomatoes when compared to WT (Additional file 6). In accordance with our results, IPT overexpression resulted in increased levels of bioactive iP, $t Z$ and their ribosides in tomato [3] and tobacco [31,32]. However, detailed data regarding all CK derivatives were not reported in these previous studies. Based on the observation that iPRMP/ iPRDP/iPRTP are the first products in CK biosynthesis $[9,10]$, that iP and iPR are the best substrates for CKX activity [34] and are in massive amounts metabolized to CK- $N$-glucoside forms (Additional file 5), we conclude that iP-type CKs have a prominent role in fast regulation and balancing of the CK metabolism.

\section{SIIPT3 and SIIPT4 participate in salinity response in tomato and Arabidopsis}

Expression analyses of SIIPT3 and SIIPT4 in tomato vegetative organs confirmed the participation of both genes in response to salt stress. After stress onset, salinity evoked repression of SIIPT3 and SIIPT4 in roots, and differently affected SIIPT3 and SIIPT4 in young and old leaves. After 6 to $8 \mathrm{~h}$ of exposure to salinity, increasing levels of both transcripts were detected in young leaves, suggesting preferential CK biosynthesis in photosynthetic organs and thus underlining CKs stress-prevention and anti-senescence properties (Figure 10B). Similarly, an augmentation of IPT expression was apparent both in maize leaves and roots following longer exposure to salinity whereas shorter salt treatment $(30 \mathrm{~min})$ induced a repression of IPT encoding genes (3 out of 4) in maize leaves [11]. Likewise, two weeks-old Arabidopsis plants showed downregulation of AtIPT1 and AtIPT3 as early as $1 \mathrm{~h}$ after salt treatment, whereas the expression of AtIPT5 and AtIPT7 was elevated. Conversely, with longer salt stress, the majority of AtIPT transcripts were repressed [35]. Together, these data indicate a dynamic regulation of CK biosynthesis/metabolism over the stress period.

Moreover, multiple putative stress-responsive cis-regulatory elements were identified in the promoter regions of BrIPTs (and $B r C K X s$ ) promoters, pointing to the response of these genes to stress stimuli and a possible response to unfavourable conditions via changing CK status [36]. Similarly in this study, an in silico analysis of SIIPT3 and SIIPT4 promoters [37] (www.dna.affrc.go.jp/PLACE/index.html) revealed the presence of putative stress response elements and many ARR binding cis-elements, indicating at least for SIIPT3 the existence of salt stress tolerance mechanisms similar to those in BrIPTs. Our findings in tomato plants illustrated that the regulation of SIIPT3 and SIIPT4 in response to salt stress is organ specific, depends on the duration of stress exposure and potentially also on the presence of cis-regulatory elements in the promoters of
CK-related genes. In addition, diverse roles of both tomato SlIPTs in salt stress responses have been characterized in Arabidopsis ipt3 $\mathrm{KO}$ complemented plants under longer period of salt treatment (Figures 4 and 5).

Transgenic plants ectopically expressing SIIPT3 under $35 S$ promoter showed superior above-ground growth after several weeks on salt medium, relative to WT plants (Figure 9A). Beneficial effects of CKs overproduction in response to salt stress were reported in tomato [3]. CKs elevation enhanced shoot growth and prevented senescence under salinity conditions due to the specific induction of IPT in roots by a heat-shock promoter (HSP70::IPT). Likewise, higher CK concentration was associated with salt tolerance in tomatoes overexpressing a cysteine-2/histidine-2-type zinc finger transcription factor [38]. Similarly, expression of Agrobacterium tumefaciens IPT under the stress inducible promoter $r d 29 A$ resulted in higher salinity tolerance in transgenic tobacco [23]. Using specific promoters for IPT overexpression also successfully improved tolerance to drought in rice [39], peanut [40], cassava [41], tobacco [42] and to the cold stress in tobacco [43]. On the other hand, tobacco plants with reduced CK contents showed smaller osmotic potential and higher drought resistance than WT plants [44]. Likewise, Arabidopsis knock-out mutants for two CK histidine kinase receptors ( $a h k 2$ and $a h k 3$ ) showed tolerance to drought and salinity [45]. Aspects of plant phenotype, such as root architecture modifications and growth inhibition in transgenic plants, influenced by imbalance between CKs accumulation and distribution can partly explain tolerance to various environmental stresses. Thus, root-specific $C K X$ overexpression enhanced root system development and consequently increased drought tolerance, nutrient uptake and leaf nutrient enrichment in Arabidopsis $[43,46]$. Consequently, it is still difficult to estimate the precise nature of CK effects due to the complexity of phytohormones metabolism and other cross-talk networks.

Salinity tolerance of 35S::SIIPT3 plants is correlated, among others, with improved nutrients balance and retention of the photosynthesis capacity, in addition to the senescence-protective effects of the photosynthetic pigments (Figure 9). Indeed, salinity strongly impacts tomato plants ionic status, as $\mathrm{Na}^{+}$concentration considerably increases in leaves, inducing a premature senescence, while $\mathrm{K}^{+}$content differently declines depending on the leaf age [4]. Transgenic tomato plants HSP70::IPT showing improved growth and final yield under long-term salinity treatment consequently accumulate less $\mathrm{Na}^{+}$in leaves and roots than WT, but higher $\mathrm{K}^{+}$contents, underlining the importance of the limitation of toxic ions accumulation for plant development [3]. In addition, salinity strongly affects plant photosynthesis, and salt-induced reduction in photosynthetic pigments such as $\mathrm{Chl}$ and $\mathrm{Car}$ have been 
reported in several studies [47]. Variations in Chl and Car contents under different salt concentrations and time exposure were observed in three varieties of tomato cultivars, suggesting that sensitivity to salt stress depends on plant genotype [48]. Our results indicate that the contents of Chl $a$ and $\mathrm{Ch} b$ as well as lutein and $\beta$-carotene are maintained in 35S::SIIPT3 plants at the same levels regardless of the growth media in contrast to WT, in which salt treatments decreased both Chl and Car contents (Figure 9B and C). Similarly, transgenic tomatoes overexpressing IPT within the root zone maintained their photosynthesis ability under long-term salinity treatement [3], while transgenic tobacco expressing stress-inducible promoter $r d 29 A-I P T$ showed only slight decrease in Chl contents in comparison to WT plants [23]. Significant accumulation of pigments involved in the xanthophyll cycle in 35S::SIIPT3 plants exposed to salt may reflect higher intensity in photoprotective function of Car in transgenic plants than in WT plants.

In summary, our results indicate that constitutive expression of SIIPT3 induces several changes in plant growth, nutrients accumulation, hormonal metabolism and photosynthesis maintenance, leading to enhanced tolerance to salt stress.

\section{Mechanism of SIIPT3 and SIIPT4 regulation under salt and CK treatments}

The comprehensive mechanism of CK regulatory feedback loop is supported by our findings that exogenous application of $t \mathrm{Z}$ inhibited the expression of SIIPT3 and SIIPT4 within $4 \mathrm{~h}$, enhanced the expression of negativefeedback regulator type-A SlARR4 and slightly moderated the expression levels of CK primary response genes type-B SlARR1 and SIARR12 (Figure 6C and D). Comparable data reporting downregulation of the IPT genes after CK treatment have been reported for Chinese cabbage [36] and maize [11]. The connection between CK homeostasis maintenance via type-B response regulators and salinity response was reported in Arabidopsis where ARR1 and ARR2 were shown to control sodium accumulation in shoots by regulating expression of genes encoding high-affinity $\mathrm{K}^{+}$transporter $1 ; 1$ responsible for $\mathrm{Na}^{+}$exclusion from the root xylem [25]. These data suggest that CK homeostasis may be effectively regulated, not only by CKs molecules, but also environmental stimuli.

Taken together, we have shown the mechanism of SIIPT3 and SIIPT4 response to early salt stress and their feedback regulation by exogenous CK treatment in tomato plants. Based on the data obtained in this study, we propose a model illustrating the complexity of $\mathrm{CK}$ networks in tomato plants in response to salinity (Additional file 8). In this model, we only included relations supported by our experimental data. We outline an organ specific downregulation of SIIPT3 and SIIPT4 transcripts immediately after stress onset, followed by transcripts increase with the duration of the stress treatment. The complexity of CK networks is demonstrated by the repression of SIIPT3 and SIIPT4 after exogenous application of $t Z$, while an overabundance of CKs (especially iP7G) in 35S::SlIPT3 may ensure a fitter phenotype under salt stress conditions. Similarly, the response to salinity by SIIPT3 and SIIPT4 was paralleled in Arabidopsis ipt3 KO complemented plants where salinity resulted in elevation of CKs (mainly $N$-glucosides) with successive downregulation of AtIPTs to maintain CK homeostasis (Figure 5, Additional file 8).

\section{Conclusions}

In summary, we have characterized SIIPT3 and SIIPT4 response to early salt stress in tomato, and identified SIIPT3 as a key player in the CK metabolism of tomato plants under salinity conditions. Our results contribute to the understanding of $\mathrm{CK}$ regulation at the molecular level and provide a potentially useful tool to obtain and improve high-quality stress-tolerant crops in agriculture.

\section{Methods}

\section{Plant materials and growth conditions}

Tomato (Solanum lycopersicum L. cv Ailsa Craig) seeds were pre-germinated on Whatman 3MM paper soaked with sterile water in a sterile petri dish until the radicle was a few $\mathrm{mm}$ long. Seeds were sown in trays filled with a perlite-vermiculite mix $(1 / 3, \mathrm{v} / \mathrm{v})$, as previously described [4]. Four weeks later, tomato plantlets cultivated in a growth chamber with a $16 \mathrm{~h}$ light $\left(24^{\circ} \mathrm{C}\right) / 8 \mathrm{~h}$ dark $\left(22^{\circ} \mathrm{C}\right)$ photoperiod were transferred into the $52 \mathrm{~L}$ tanks containing aereted half-strength modified Hoagland nutrient solution, and kept in hydroponic system for an additional ten days before applying the salt stress, as previously described [38].

Salt stress treatment of wild-type tomatoes was evoked by direct addition of $150 \mathrm{mM} \mathrm{NaCl}$ into the culture tank. For hormonal assays, plants were grown in smaller tanks with a capacity of $4.5 \mathrm{~L}$. Zeatin (mixed cis- and transisomers including approximately $80 \%$ of trans-zeatin, Sigma Z0164) was used at a final concentration of 10 $\mu \mathrm{M}$. In order to investigate the spatio-temporal expression profiles of SIIPT3 and SIIPT4, plants were grown in soil in the greenhouse during 8 weeks. Three plants were collected for each time point of the experiment $(0 \mathrm{~h}, 1 \mathrm{~h}, 2 \mathrm{~h}, 4 \mathrm{~h}, 8 \mathrm{~h}$ and $12 \mathrm{~h})$. After harvesting, samples were immediately frozen in liquid nitrogen and stored at $-80^{\circ} \mathrm{C}$ until further analyses. For tomato $35 \mathrm{~S}::$ SIIPT3 (L1) in vitro culture, seeds were surface sterilized with a mixture of $2.5 \%(\mathrm{v} / \mathrm{v})$ potassium hypochlorite and $0.02 \%(\mathrm{v} / \mathrm{v})$ Triton X-100 for $15 \mathrm{~min}$ and rinsed 5 times with sterile water. Seeds were sown on full MS 
media and the medium for transgenic line selection was supplemented with kanamycin $(100 \mathrm{mg} / \mathrm{L})$. Two weeks later, plants were transferred into magenta boxes on the control medium and the medium containing $\mathrm{NaCl}(100 \mathrm{mM})$ and cultivated under the same conditions as before $\left(16 \mathrm{~h}\right.$ light $\left(24^{\circ} \mathrm{C}\right) / 8 \mathrm{~h}$ dark $\left(22^{\circ} \mathrm{C}\right)$ photoperiod in the growth chamber). Phenotypic characteristics were evaluated after four weeks.

Arabidopsis thaliana ecotype Columbia was used as the wild-type for all Arabidopsis experiments. The ipt3 T-DNA insertion line N553810 was obtained from the Nottingham Arabidopsis Stock Center. Seeds were surface sterilized as described for tomato and kept at $4^{\circ} \mathrm{C}$ for $48 \mathrm{~h}$ before being sown on Petri dishes containing half-strength MS with $0.9 \%(\mathrm{w} / \mathrm{v})$ agar. Additionally, the medium for ipt $3 \mathrm{KO}$ mutants selection contained $35 \mathrm{mg} / \mathrm{L}$ kanamycin and SIIPT3 and SIIPT4 Arabidopsis complemented plants were selected on the medium supplemented with $15 \mathrm{mg} / \mathrm{L}$ hygromycin. Plates were then transferred to the growth chamber with a $16 \mathrm{~h}$ light $\left(20^{\circ} \mathrm{C}\right) / 8 \mathrm{~h}$ dark $\left(18^{\circ} \mathrm{C}\right)$ photoperiod. For the analysis of salt stress tolerance, halfstrength MS medium containing $100 \mathrm{mM} \mathrm{NaCl}$ was used. The root length (from hypocotyl base to the root tip) of at least twenty vertically growing ten-days-old plants was measured. Differences in root length were tested for significance using a Mann-Whitney $U$ test and data representing two biological replicates. Germination percentage was determined for eighteen seedlings after 24 $\mathrm{h}$ using a magnifying glass, when the radicle occurs. Survival percentage of eighteen seedlings was evaluated in thirteen-day-old plants. Total numbers of germinated and non-germinated seeds (54) were cross tabulated for each pair of groups and Fisher's exact test was then applied to the resulting $2 \times 2$ contingency tables. For both assays, the data represent three biological replicates.

\section{Plasmid constructions and plant transformation}

SIIPT3, SIIPT4, SlARR1 (XP_004239797), SlARR4 (XP_ 004238726) and SlARR12 (XP_004251765) cDNAs were amplified with the $P f u$ DNA polymerase (Promega) in a final reaction volume of $50 \mu \mathrm{L}$ following the manufacturer's instructions, using $300 \mathrm{ng}$ of cDNAs synthesized from total RNA extracted from tomato grown in vitro as template. Specific primers used to amplify the coding sequence of SIIPT3 and SIIPT4 contain anchors: forward (5'-GGGGACAAGTTTGTACAAAAAAGCAGGCT-3') and reverse (5'-GGGGACCACTTTGTACAAGAAAGC TGGGT-3') allowing BP cloning (Invitrogen) in the pDONR221 vector. Specific primers for every coding sequence were as follows: IPT3F (5'-TATGAATATTGT GTTACAACATATTG-3'), IPT3R (5' -CTAGTGCGTCA TAGTAGCAAC-3'), IPT4F (5'-ATGATTGGCATGATG AACTCT-3'), IPT4R (5' -TTAATAGTGAGATGCTGCT
GCC-3'). The PCR product was then transferred to the pDONR221 entry vector (Invitrogen) by a BP recombination reaction prior to DNA sequencing (Macrogen). SIIPT3 and SIIPT4 were subsequently transferred either to the pK7WG2D or to the pH7WG2D binary vectors [49]. SlARR coding sequences were amplified with the following primers: ARR1F (5' -AAAAAATTCAAAATTT TGAAGAAAGT-3') and ARR1R (5'-AACTACGCTCT TTTCGCATC-3'), ARR4F (5'-ATTTGGTGAAATATT TGTGGGTT-3') and ARR4R (5'-GCTTAAACGACCC CGGAAGTA-3'), ARR12F (5'-ATGACTGTGGAGGAA ATTAGA-3') and ARR12R (5'-TCATAAACCTGAACC AAGTGAA-3'), prior to cloning into the pGEM-T-easy vector (Promega).

SIIPT3 and SIIPT4 constructs were introduced into the Agrobacterium tumefaciens strain GV3101 for Arabidopsis transformation and in the LBA44A4 for tomato transformation. Competent cells were prepared as previously described [50]. For subcellular localization, SIIPT3 and SIIPT4 cDNA sequences lacking a stop codon were cloned in frame with the GFP sequence in the pK7FWG2 binary vector [49].

Tomato stable transformation was adapted from a published method [51] and tomato genomic DNA extraction was performed as described before [52].

Arabidopsis plants were transformed by the floral dip method [53]. For Arabidopsis ipt3 complementation, a combination of 3 primers LBb1.3 (5' ${ }^{\prime}$-ATTTTGCCGAT TTCGGAAC-3'), IPT3LP (5'-TGGAATGGTTGAGGA AGTCAG-3'), IPT3RP (5'-CATTGGCTTAGAAATTT GTGTCC-3') was used to identify homozygous plants in the segregating F3 population.

\section{RNA extraction and real-time PCR analysis}

Tomato total RNAs were extracted from all tissues, treated with the RNase-free DNase I (Promega) and purified according to a previous reference [54]. An aliquot of 2 $\mu \mathrm{g}$ was subsequently used as a template for reversetranscription (RevertAid ${ }^{\mathrm{Tx}} \mathrm{H}$ Minus First Strand cDNA Synthesis Kit, Fermentas) using oligo $\mathrm{d}(\mathrm{T})_{18}$ according to the instructions in the manual. Transcript levels of the different genes were measured by qRT-PCR using SYBR Green on a LightCycler 480 II (Roche). PCR reactions were performed in triplicate using $0.2 \mu \mathrm{M}$ of each primer, $5 \mu \mathrm{L}$ SYBR Green mix (Promega), and $300 \mathrm{ng}$ of DNAse-treated cDNA in a final volume of $10 \mu \mathrm{L}$. Negative controls were included in each run. PCR conditions were: initial denaturation at $95^{\circ} \mathrm{C}$ for $120 \mathrm{~s}$ followed by 45 cycles of $95^{\circ} \mathrm{C}$ for $10 \mathrm{~s}$, and $58^{\circ} \mathrm{C}$ for $15 \mathrm{~s}$. Amplification was followed by melting curve analysis to check the specificity of each reaction. The primers IPT3PCRQF $\left(5^{\prime}\right.$-CCTTCT TGCACAAAGTTGCT-3') and IPT3PCRQR (5' -TGAGG TTATTGATATTAGCAAATA-3') were used to amplify a 
107 bp sequence, while IPT4PCRQF (5' -GGACAGAGCA GAAAGAT-3') and IPT4PCRQR (5'-TAATAGTGAGAT GCTGCTGCCA-3') allowed amplification a 108 bp PCR product.

Data obtained from tomato were normalized according to SlGAPDH and SlActin expression levels. The primers used were GAPDHF (5'-GGTGCCAAGAAGGTTGTG AT-3') and GAPDHR (5' -TTTTCTGGGTGGCAGTCA T-3') that generated a 217 bp PCR product, ActinF (5'-ATGGTGGGTATGGGTCAAAA-3') and ActinR (5'-GAGGACAGGATGCTCCTCAG-3') that allowed the formation of a $183 \mathrm{bp}$ PCR product. Normalized expression of SIIPT3 and SIIPT4 was calculated using the Gene Expression Analysis for iCycle iQ_ Real Time PCR Detection System software from Bio-Rad with a method derived from the algorithms previously outlined [55].

A similar procedure was adopted for the qRT-PCR analysis of Arabidopsis samples. The list of primers used for both tomato and Arabidopsis qRT-PCR analysis is attached as Supplementary data (Additional files 9 and 10). Statistical differences in target gene transcripts were evaluated using unpaired Student's $t$-test $\mathrm{p} \leq 0.05$ to compare $\Delta \mathrm{Ct}$ values.

\section{In vitro isopentenyltransferase activity assay}

SIIPT3 and SIIPT4 coding sequence were respectively amplified by PCR with the following primers: SIIPT3F (5' - GGATCCTAATACGACTCACTATAGGGAACAG CCACCATGAATATTGTGTTACAACATATT-3') and SIIPT3R (5'-TTTTTTTTTTTTTTTTTTTTTTTTTT TTTTTTAGTGCGTCATAGTAGCAA-3') SIIPT4F (5'GGATCCTAATACGACTCACTATAGGGAACAGCCAC CATGATTGGCATGATGAACTCT -3'), SIIPT4R (5'TTTTTTTTTTTTTTTTTTTTTTTTTTTTTTTTAAT AGTGAGATGCTGCTG-3'). The forward primer included a sequence of the T7 RNA polymerase promoter, while the reverse primer contained a stop codon and a polyadenylated extremity. Generated amplicons were subcloned into pGEMT-T Easy vector (Promega) before sequencing. $800 \mathrm{ng}$ of purified PCR product were used for in vitro protein translation in rabbit reticulocyte cells (TnT ${ }^{\oplus}$ T7 Quick Coupled Transcription/Translation System, Promega), following the manufacturer's instructions, in a final reaction volume of $50 \mu \mathrm{L}$.

SIIPT3 and SIIPT4 were examined for DMAPP: $\left[{ }^{3} \mathrm{H}\right]$ AMP, DMAPP: $\left[{ }^{3} \mathrm{H}\right] \mathrm{ADP}$ and DMAPP: $\left[{ }^{3} \mathrm{H}\right] \mathrm{ATP}$ isopentenyltransferase activity using an adapted method $[9,32]$. The reaction mixture, consisting of $50 \mu \mathrm{L}$ protein produced in vitro as described above, $100 \mu \mathrm{L}$ of salts and buffer containing $37.50 \mathrm{mM} \mathrm{KCl}, 5 \mathrm{mM}$ $\mathrm{MgCl}_{2}$ and $12.5 \mathrm{mM}$ Tris- $\mathrm{HCl}(\mathrm{pH} 7.5)$ and $100 \mu \mathrm{L}$ of substrates DMAPP $(34 \mathrm{nmol})$ plus $\left[{ }^{3} \mathrm{H}\right]$ AMP, $\left[{ }^{3} \mathrm{H}\right] \mathrm{ADP}$ or $\left[{ }^{3} \mathrm{H}\right] \mathrm{ATP}$ (each $1 \mathrm{nmol}, 20 \mathrm{Ci} / \mathrm{mmol}$ ) was incubated at $30^{\circ} \mathrm{C}$ for $1 \mathrm{~h}, 2 \mathrm{~h}, 8 \mathrm{~h}, 24 \mathrm{~h}$ and $48 \mathrm{~h}$. Negative controls included either a crude extract of non-transformed rabbit reticulocyte cells or no cells at all. To stop the reaction and to precipitate the SIIPT3 or SIIPT4 proteins, cold ethanol $(150 \mu \mathrm{L})$ was added into an aliquot of $50 \mu \mathrm{L}$ of reaction mixture at the indicated times. The samples were vortexed and stored at $-20^{\circ} \mathrm{C}$ for $2 \mathrm{~h}$. The mixture was then centrifuged for $25 \mathrm{~min}, 20000 \mathrm{~g}$ at $4^{\circ} \mathrm{C}$. The supernatant was recovered in clean tubes and evaporated in a vacuum concentrator (Alpha RVC, Christ) to dryness. The pellet was resuspended in $50 \mu \mathrm{L}$ of $5 \% \mathrm{MeOH}$. Each sample was analyzed by HPLC (Perkin Elmer) coupled to a radioactivity flow detector (Ramona 2000, Raytest). The radioactive metabolites were identified on the basis of comparison of their retention times with authentication standards. IPT activity was determined in four independent experiments, which showed the same tendencies although with different absolute values (Additional files 11). Therefore the results of one representative experiment are presented.

\section{Cytokinins extraction and quantification}

Endogenous CKs were extracted from homogenized young fully expanded tomato leaves ( $15 \mathrm{mg}$ of dry weight) and 17 DAS Arabidopsis plants (150 mg of fresh weight) using an extraction buffer consisting of methanol/formic acid/water (15/1/4, v/v/v) according to the published method [56]. Following the addition of stable isotope labeled internal standards (10 pmols), samples were extracted for $1 \mathrm{~h}$ at $-20^{\circ} \mathrm{C}$. The solids were separated by centrifugation $\left(20000 \mathrm{~g}, 20 \mathrm{~min}, 4^{\circ} \mathrm{C}\right)$ with a subsequent collection of supernatants. The pellets were re-extracted with an additional $0.5 \mathrm{~mL}$ of extraction buffer $(30 \mathrm{~min}$ at $\left.-20^{\circ} \mathrm{C}\right)$. The supernatants were collected after recentrifugation, and incubated $30 \mathrm{~min}$ at $-80^{\circ} \mathrm{C}$. Samples were evaporated in a vacuum concentrator (Alpha RVC, Christ), re-dissolved in $0.5 \mathrm{~mL}$ of $1 \mathrm{M}$ formic acid and applied to a mixed mode reversed phase-cation exchange SPE column (Oasis-MCX, Waters).

The CK fraction was sequentially eluted with $0.35 \mathrm{M}$ $\mathrm{NH}_{4} \mathrm{OH}$ in $60 \%$ methanol. This fraction was evaporated to dryness in a vacuum concentrator and dissolved in $5 \% \mathrm{MeOH}$. An aliquot $(0.01 \mathrm{~mL})$ from each sample was separately analyzed on a high-performance liquid chromatography (HPLC) (Ultimate 3000, Dionex) coupled to a hybrid triple quadrupole/linear ion trap mass spectrometer (3200 Q TRAP, Applied Biosystems) using a multilevel calibration graph with $\left[{ }^{2} \mathrm{H}\right]$-labeled internal standards as described before $[57,58]$. Data are presented as mean \pm standard error.

\section{CKX activity assay in vitro}

CKX enzymes were extracted and partially purified from the same tomato leaves samples used for hormones 
extraction (WT and 35S::SlIPT3) according to a published reference [59]. CKX activity was determined by in vitro assays based on the conversion of $\left[2-{ }^{3} \mathrm{H}\right] \mathrm{iP}$ (prepared by the Isotope Laboratory, IEB ASCR, Prague, Czech Republic) to $\left[{ }^{3} \mathrm{H}\right]$ adenine and expressed as pmol adenine $\mathrm{mg}$ protein $^{-1} \mathrm{~h}^{-1}$. The CKX activity was determined in two biological replicates for each variant.

\section{Photosynthetic pigments and ion extraction, quantification and analysis}

The content of photosynthetic pigments (Chl $a$ and $\mathrm{Chl}$ $b$, $\beta$-carotene, lutein, neoxanthin, violaxanthin, zeaxanthin and antheraxanthin) was determined in acetone extracts made from the lyophilized developing leaves of 4 weeks-old plants analyzed by a high-performance liquid chromatography (ECOM, Czech Republic). The analysis was made using a reversed phase column (Watrex Nucleosil $1205 \mathrm{C} 18,5 \mu \mathrm{m}$ particle size, $125 \times 4 \mathrm{~mm}$, ECOM, Czech Republic). The solvent system comprised acetonitrile/methanol/water $(80 / 12 / 10, \mathrm{v} / \mathrm{v} / \mathrm{v})$ followed by methanol/ethylacetate $(95 / 5, \mathrm{v} / \mathrm{v})$, the total analysis time was $25 \mathrm{~min}$, and the linear gradient was run from 2 to $6 \mathrm{~min}$ (the flow rate was $1 \mathrm{~cm}^{3} \mathrm{~min}^{-1}$, the detection wavelength was $445 \mathrm{~nm}$ ). Data were captured and calculated by PC-software Clarity (DataApex, Czech Republic). The photosynthetic pigments were determined in two biological replicates of each variant measured independently by two times.

Shoot mineral quantification was conducted on the same samples as for pigments analysis using an atomic absorption spectrometer (Thermo Scientific ICE3300) as described previously [38].

\section{Availability of supporting data}

Phylogenetic data are available in Treebase (www.tree base.org) database under submission identity 16841.

\section{Additional files}

Additional file 1: Phenotype of 5 DAS Arabidopsis WT, ipt3 KO, and SIIPT3 or SIIPT4 complemented plants cultivated on control medium. Additional file 2: Endogenous CKs content (pmol/g FW) of 17 DAS Arabidopsis ipt3 plants complemented with SIIPT3 or SIIPT4 and grown on control medium. The system of abbreviations was adopted and modified according to published reference [60].

Additional file 3: Endogenous CKs content (pmol/g FW) of 17 DAS Arabidopsis ipt3 plants complemented with SIIPT3 or SIIPT4 and grown on salt medium $(100 \mathrm{mM} \mathrm{NaCl})$. The system of abbreviations was adopted and modified according to published reference [60].

Additional file 4: Phenotype of T1-generation tomatoes 35::SIIPT3 Line 1 (L1) transformants.

Additional file 5: Endogenous CKs content (pmol/g FW) in young leaves of T0 35S::SIIPT3 tomatoes. The system of abbreviations was adopted and modified according to published reference [60].
Additional file 6: Cytokinin oxidase/dehydrogenase (CKX) activity in 35S::SIIIPT3 tomato young leaves ( $\mathrm{L} 6$ and L7).

Additional file 7: The content of photosynthetic pigments ( $\mu \mathrm{g} / \mathrm{g} \mathrm{DW}$ ) in 4 weeks-old tomato developing leaves of WT and T2-generation of 35S::SIIIPT3 (L1) tomato plants cultivated on the control and salt growth media. DEPS (De-epoxidation state) is calculated according to formula: DEPS $=\left(0.5^{*} \mathrm{~A}+\mathrm{Z}\right) /(\mathrm{V}+\mathrm{A}+\mathrm{Z})$; DEPS (De-epoxidation state per chlorophyll) is calculated according to formula: DEPSC $=\left(0.5^{*} \mathrm{~A}+\mathrm{Z}\right) /(\mathrm{Chl} a+b)$. $\wedge$ expressed in relative unit. *Data represent means and SD of two replicates. *significantly different datasets from WT (unpaired Student's $t$-test, $P \leq 0.05$ ).

Additional file 8: Hypothetical scheme of SIIPT3 and SIIPT4 response to early salt stress and the feedback regulation by exogenous CK treatment in tomato plants. After salt stress (150 mM $\mathrm{NaCl}$ ) treatment, immediate down-regulation of SIIPT3 and SIIPT4 transcripts with a subsequent up-regulation in tomato vegetative organs was determined. Repression of both genes after exogenous application of $t Z$ demonstrated the complexity of CK networks, while the overabundance of CKs (specially iP7G) in 355:-SIIPT3 may ensure a stronger phenotype under salt stress (100 mM NaCl) conditions.

Additional file 9: Sequences of tomato primers used for qRT-PCR analysis.

Additional file 10: Sequences of Arabidopsis primers used for qRT-PCR analysis.

Additional file 11: In vitro enzymatic activity of SIIPT3 and SIIPT4. Conversion of non-labeled AMP added into the water and cells containing SIIPT3 (A) or cells containing SIIPT4 (B) measured by IP-HPLC with UV detector. Conversion of radioactive labeled precursors AMP, ADP and ATP added into the water and cells containing SIIPT3 or SIIPT4 were measured by LCMS (C).

\section{Competing interests}

The authors declare that they have no competing interests.

\section{Authors' contributions}

$\mathrm{EZ}$ and $\mathrm{IH}$ designed the experiments and wrote the manuscript. $\mathrm{KH}$ drafted the manuscript and helped with figure preparation. PID and YM performed or assisted in the execution of specific experiments. PH helped with the statistical analyses. DH and DP provided help with photosynthetic pigments analyses. VM and SL guided the study and revised the manuscript. All authors read and approved the manuscript.

\section{Acknowledgements}

The authors thank Dr Miroslav Kamínek for critical reading of manuscript, Dr Said Hafidh and Dr James Stones for language editing and Marie Korecká for excellent technical support. We also thank Abdelmounaim Errachid for his help with confocal microscope, and Dr Etienne G. Danchin for his help with phylogenetic analyses.

This work was supported by the Czech Science Foundation [P506/11/0774]; the Fonds National de la Recherche [FNRS; convention $n^{\circ} 1.5117 .11$ ]; and the Wallonie-Bruxelles International program [Rhéa 2011/35047 and WBI/14-3].

\section{Author details}

${ }^{1}$ Institute of Experimental Botany, Academy of Sciences of the Czech Republic, Prague 165 02, Czech Republic. ²Département Sciences du vivant, Centre wallon de Recherches Agronomiques, Gembloux B-5030, Belgium. ${ }^{3}$ Department of Biomedical Informatics, Faculty of Biomedical Engineering, Czech Technical University in Prague, Kladno 272 01, Czech Republic. ${ }^{4}$ Groupe de Recherche en Physiologie Végétale (GRPV), Earth and Life Institute - Agronomy (ELI-A), Université catholique de Louvain (UCL), Louvain-la-Neuve 1348, Belgium. Institut National de la Recherche Agronomique, Institut Sophia Agrobiotech (ISA), UMR INRA 1355, CNRS 7254, Université de Nice-Sophia Antipolis, 400 route des Chappes, BP167, F-06903 Sophia-Antipolis Cedex, France. 


\section{References}

1. Cuartero J, Fernández-Muñoz R. Tomato and salinity. Sci Horticult. 1999;78:83-125.

2. Albacete A, Ghanem ME, Martínez-Andújar C, Acosta M, Sánchez-Bravo J, Martínez $V$, et al. Hormonal changes in relation to biomass partitioning and shoot growth impairment in salinized tomato (Solanum lycopersicum L.) plants. J Exp Bot. 2008;59:4119-31.

3. Ghanem ME, Albacete A, Smigocki AC, Frébort I, Pospísilová H, Martínez-Andújar $C$, et al. Root-synthesized cytokinins improve shoot growth and fruit yield in salinized tomato (Solanum lycopersicum L.) plants. J Exp Bot. 2011;62:125-40.

4. Ghanem ME, Albacete A, Martínez-Andújar C, Acosta M, Romero-Aranda R, Dodd IC, et al. Hormonal changes during salinity-induced leaf senescence in tomato (Solanum lycopersicum L.). J Exp Bot. 2008;59:3039-50.

5. Cueno ME, Imai K, Ochiai K, Okamoto T. Cytokinin dehydrogenase differentially regulates cytokinin and indirectly affects hydrogen peroxide accumulation in tomato leaf. J Plant Physiol. 2012;169:834-8.

6. Spíchal L. Cytokinins - recent news and views of evolutionally old molecules. Funct Plant Biol. 2012;39:267-84.

7. Mok DW, Mok MC. Cytokinin metabolism and action. Annu Rev Plant Physiol Plant Mol Biol. 2001;52:89-118.

8. Gajdošová S, Spíchal L, Kamínek M, Hoyerová K, Novák O, Dobrev PI, et al. Distribution, biological activities, metabolism, and the possible function of cis-zeatin-type cytokinins in plants. J Exp Bot. 2011;62:2827-40.

9. Kakimoto T. Identification of plant cytokinin biosynthetic enzymes as dimethylallyl diphosphate:ATP/ADP isopentenyltransferases. Plant Cell Physiol. 2001;42:677-85.

10. Takei K, Sakakibara H, Sugiyama T. Identification of genes encoding adenylate isopentenyltransferase, a cytokinin biosynthesis enzyme, in Arabidopsis thaliana. J Biol Chem. 2001;276:26405-10.

11. Vyroubalová S, Václavíková K, Turecková V, Novák O, Smehilová M, Hluska T, et al. Characterization of new maize genes putatively involved in cytokinin metabolism and their expression during osmotic stress in relation to cytokinin levels. Plant Physiol. 2009;151:433-47.

12. Matsuo S, Kikuchi K, Fukuda M, Honda I, Imanishi S. Roles and regulation of cytokinins in tomato fruit development. J Exp Bot. 2012;63:5569-79.

13. Smigocki AC, Owens LD. Cytokinin gene fused with a strong promoter enhances shoot organogenesis and zeatin levels in transformed plant cells. Proc Natl Acad Sci USA. 1988;85:5131-5.

14. Smart CM, Scofield SR, Bevan MV, Dyer TA. Delayed leaf senescence in tobacco plants transformed with tmr, a gene for cytokinin production in Agrobacterium. Plant Cell. 1991;3:647-56.

15. Ebinuma $H$, Sugita K, Matsunaga E, Yamakado M. Selection of marker-free transgenic plants using the isopentenyl transferase gene. Proc Natl Acad Sci USA. 1997:94:2117-21.

16. Sýkorová B, Kurešová G, Daskalova S, Trčková M, Hoyerová K, Raimanová I, et al. Senescence-induced ectopic expression of the A. tumefaciens ipt gene in wheat delays leaf senescence, increases cytokinin content, nitrate influx, and nitrate reductase activity, but does not affect grain yield. J Exp Bot. 2008:59:377-87.

17. Miyawaki K, Tarkowski P, Matsumoto-Kitano M, Kato T, Sato S, Tarkowska D, et al. Roles of Arabidopsis ATP/ADP isopentenyltransferases and tRNA isopentenyltransferases in cytokinin biosynthesis. Proc Nat Acad Sci USA. 2006:103:16598-603.

18. $\mathrm{Ma} \mathrm{QH}$. Genetic engineering of cytokinins and their application to agriculture. Crit Rev Biotechnol. 2008;28:213-32.

19. Peleg Z, Apse MP, Blumwald E. Engineering salinity and water-stress tolerance in crop plants: getting closer to the field. Adv Bot Res. 2011;57:405-28.

20. Zalabák D, Pospíšilová H, Šmehilová M, Mrízová K, Galuszka P. Genetic engineering of cytokinin metabolism: prospective way to improve agricultural traits of crop plants. Biotechnol Adv. 2013;31:97-117.

21. Nishiyama R, Le DT, Watanabe Y, Matsui A, Tanaka M, Seki M, et al. Transcriptome analyses of a salt-tolerantcytokinin-deficient mutant reveal differential regulation of salt stress response by cytokinin deficiency. PLoS One. 2012;7:e32124.

22. Liu YD, Yin ZJ, Yu JW, Li J, Wei HL, Han XL, et al. Improved salt tolerance and delayed leaf senescence in transgenic cotton expressing the Agrobacterium IPT gene. Biol Plant. 2012;56:237-46.

23. Qiu W, Liu M, Qiao G, Jiang J, Xie L, Zhuo R. An isopentenyl transferase gene driven by the stress-inducible rd29A promoter improves salinity stress tolerance in transgenic tobacco. Plant Mol Biol Rep. 2012;30:519-28
24. Hwang I, Sheen J, Müller B. Cytokinin signaling networks. Annu Rev Plant Biol. 2012:63:353-80.

25. Mason MG, Jha D, Salt DE, Tester M, Hill K, Kieber JJ, et al. Type-B response regulators $A R R 1$ and $A R R 12$ regulate expression of AtHKT1;1 and accumulation of sodium in Arabidopsis shoots. Plant J. 2010;64:753-63.

26. The Tomato Genome Consortium. The tomato genome sequence provides insights into fleshy fruit evolution. Nature. 2012;485:635-41.

27. Tamura K, Peterson D, Peterson N, Stecher G, Nei M, Kumar S. MEGA5: Molecular evolutionary genetics analysis using maximum likelihood, evolutionary distance, and maximum parsimony methods. Mol Biol Evol. 2011;28:2731-9.

28. Nakai K, Horton P. PSORT: a program for detecting sorting signals in proteins and predicting their subcellular localization. Trends Biochem Sci. 1999;24:34-6.

29. Kasahara H, Takei K, Ueda N, Hishiyama S, Yamaya T, Kamiya Y, et al. Distinc isoprenoid origins of cis- and trans-zeatin biosyntheses in Arabidopsis. J Biol Chem. 2004;279:14049-54.

30. Galichet A, Hoyerová K, Kamínek M, Gruissem W. Farnesylation directs AtIPT3 subcellular localization and modulates cytokinin biosynthesis in Arabidopsis. Plant Physiol. 2008;146:1155-64

31. Zhu YD, Jin YS, Wei S, Li H, Zhang W. Functional analysis of the isopentenyltransferase gene MdIPT3a from apple (Malus pumila Mill.). J Horticultural Sci. Biotech. 2012;87:478-84.

32. Brugière $\mathrm{N}$, Humbert $\mathrm{S}$, Rizzo N, Bohn J, Habben JE. A member of the maize isopentenyl transferase gene family, Zea mays isopentenyl transferase 2 (ZmIPT2), encodes a cytokinin biosynthetic enzyme expressed during kernel development. Cytokinin biosynthesis in maize. Plant Mol Biol. 2008;67:215-29.

33. Medford JI, Horgan R, El-Sawi Z, Klee HJ. Alterations of endogenous cytokinins in transgenic plants using a chimeric isopentenyl transferase gene. Plant Cell. 1989:1:403-13.

34. Armstrong DJ. Cytokinin oxidase and the regulation of cytokinin degradation. In: Mok DWS, Mok MC, editors. Cytokinins: Chemistry, Activity and the Function. Boca Raton, FL: CRC Press; 1994. p. 139-54.

35. Nishiyama R, Watanabe $Y$, Fujita $Y$, Le DT, Kojima M, Werner T, et al. Analysis of cytokinin mutants and regulation of cytokinin metabolic genes reveals important regulatory roles of cytokinins in drought, salt and abscisic acid responses, and abscisic acid biosynthesis. Plant Cell. 2011;23:2169-83.

36. Liu Z, LV Y, Zhang M, Liu Y, Kong L, Zou M, et al. Identification, expression, and comparative genomic analysis of the IPT and CKX gene families in Chinese cabbage (Brassica rapa ssp. pekinensis). BMC Genomics. 2013;14:594.

37. Higo K, Ugawa Y, Iwamoto M, Korenaga T. Plant cis-acting regulatory DNA elements (PLACE) database:1999. Nucleic Acids Res. 1999;27:297-300

38. Hichri I, Muhovski Y, Žižkova E, Dobrev PI, Franco-Zorilla JM, Solano R, et al. The Solanum lycopersicum Zinc Finger2 cysteine-2/histidine-2 repressor-like transcription factor regulates development and tolerance to salinity in tomato and Arabidopsis. Plant Physiol. 2014;164:1967-90.

39. Peleg Z, Reguera M, Tumimbang E, Walia H, Blumwald E. Cytokinin mediated source/sink modifications improve drought tolerance and increase grain yield in rice under water-stress. Plant Biotech J. 2011;9:747-58.

40. Qin H, Gu Q, Zhang J, Sun L, Kuppu S, Zhang Y, et al. Regulated expression of an isopentenyltransferase gene (IPT) in peanut significantly improves drought tolerance and increases yield under field conditions. Plant Cell Physiol. 2011;52:1904-14

41. Zhang $P$, Wang WQ, Zhang GL, Kaminek M, Dobrev P, Xu J, et al. Senescence-inducible expression of isopentenyl transferase extends leaf life, increases drought stress resistance and alters cytokinin metabolism in cassava. J Integr Plant Biol. 2010;52:653-69.

42. Rivero RM, Kojima M, Gepstein A, Sakakibara H, Mittler R, Gepstein S, et al. Delayed leaf senescence induces extreme drought tolerance in a flowering plant. Proc Natl Acad Sci USA. 2007;104:19631-6.

43. Guo JC, Duan RJ, Hu XW, Li KM, Fu SP. Isopentenyl transferase gene (ipt) downstream transcriptionally fused with gene expression improves the growth of transgenic plants. Transgenic Res. 2010;19:197-209.

44. Macková H, Hronková M, Dobrá J, Turečková V, Novák O, Lubovská Z, et al. Enhanced drought and heat stress tolerance of tobacco plants with ectopically enhanced cytokinin oxidase/dehydrogenase gene expession. J Exp Bot. 2013;64:2805-15. 
45. Tran LS, Urao T, Qin F, Maruyama K, Kakimoto T, Shinozaki K, et al. Functional analysis of AHK1/ATHK1 and cytokinin receptor histidine kinases in response to abscisic acid, drought, and salt stress in Arabidopsis. Proc Natl Acad Sci USA. 2007:104:20623-8.

46. Werner T, Nehnevajova E, Köllmer I, Novák O, Strnad M, Krämer U, et al. Root-specific reduction of cytokinin causes enhanced root growth, drought tolerance, and leaf mineral enrichment in Arabidopsis and tobacco. Plant Cell. 2010;22:3905-20.

47. Ashraf M, Harris PJC. Photosynthesis under stressful environments: an overview. Photosynthetica. 2013;51:163-90.

48. Doganlar ZB, Demir K, Basak H, Gul I. Effects of salt stress on pigment and total soluble protein contents of three different tomato cultivars. Afr J Agric Res. 2010;5:2056-65.

49. Karimi M, Inzé D, Depicker A. GATEWAY vectors for Agrobacterium-mediated plant transformation. Trends Plant Sci. 2002;7:193-5.

50. Jyothishwaran G, Kotresha D, Selvaraj T, Srideshikan SM, Rajvanshi PK, Jayabaskaran C. A modified freeze-thaw method for efficient transformation of Agrobacterium tumefaciens. Current Sci. 2007;93:770-2.

51. Ellul P, Garcia-Sogo B, Pineda B, Ríos G, Roig LA, Moreno V. The ploidy level of transgenic plants in Agrobacterium-mediated transformation of tomato cotyledons (Lycopersicon esculentum Mill.) is genotype and procedure dependent. Theor Appl Genet. 2003;106:231-8.

52. Fulton TM, Chunwongse J, Tanksley SD. Microprep protocol for extraction of DNA from tomato and other herbaceous plants. Plant Mol Biol Rep. 1995;13:207-9

53. Clough SJ, Bent AF. Floral dip: a simplified method for agrobacterium-mediated transformation of Arabidopsis thaliana. Plant J. 1998;16:735-43.

54. Hichri I, Heppel SC, Pillet J, Léon C, Czemmel S, Delrot S, et al. The basic helix-loop-helix transcription factor MYC1 is involved in the regulation of the flavonoid biosynthesis pathway in grapevine. Mol Plant. 2010;3:509-23.

55. Vandesompele J, De Preter K, Pattyn F, Poppe B, Van Roy N, De Paepe A, et al. Accurate normalization of real-time quantitative RT-PCR data by geometric averaging of multiple internal control genes. Genome Biol. 2002;3:Research0034.

56. Dobrev PI, Kamínek M. Fast and efficient separation of cytokinins from auxin and abscisic acid and their purification using mixed-mode solid-phase extraction. J Chromatogr A. 2002;950:21-9.

57. Dobrev PI, Vankova R. Quantification of abscisic acid, cytokinin, and auxin content in salt-stressed plant tissues. In: Shabala S, Cuin TA, editors. Plant salt tolerance: methods and protocols. Methods in molecular biology, vol. 913. New York: Springer Science + Business Media; 2012. p. 251-61.

58. Djilianov DL, Dobrev PI, Moyankova DP, Vankova R, Georgieva DT, Gajdošová S, et al. Dynamics of endogenous phytohormones during desiccation and recovery of the resurrection plant species Haberlea rhodopensis. J Plant Growth Regul. 2013;32:564-74.

59. Motyka V, Vaňková R, Čapková V, Petrášek J, Kamínek M, Schmülling T. Cytokinin-induced upregulation of cytokinin oxidase activity in tobacco includes changes in enzyme glycosylation and secretion. Physiol Plant. 2003;117:11-21.

60. Kamínek M, Březinová A, Gaudinová A, Motyka V, Vaňková R, Zažímalová E. Purine cytokinins: a proposal of abbreviations. Plant Growth Regul. 2000;32:253-6.

\section{Submit your next manuscript to BioMed Central and take full advantage of:}

- Convenient online submission

- Thorough peer review

- No space constraints or color figure charges

- Immediate publication on acceptance

- Inclusion in PubMed, CAS, Scopus and Google Scholar

- Research which is freely available for redistribution 